Tropical Journal of Pharmaceutical Research July 2018; 17 (7): 1397-1406

ISSN: 1596-5996 (print); 1596-9827 (electronic)

(1) Pharmacotherapy Group, Faculty of Pharmacy, University of Benin, Benin City, 300001 Nigeria.

Available online at http://www.tjpr.org

Original Research Article

http://dx.doi.org/10.4314/tjpr.v17i7.25

\title{
Synthesis of 3-[4-(2-furoyl)-1-piperazinyl]- $N$ - (substituted)propanamides as promising antibacterial agents with mild cytotoxicity
}

\author{
Ghulam Hussain ${ }^{1}$, Muhammad A Abbasi ${ }^{1 \star}$, Aziz-ur-Rehman', Sabahat Z \\ Siddiqui ${ }^{1}$, Irshad Ahmad ${ }^{2}$, Rabia Malik ${ }^{2}$, Muhammad Shahid ${ }^{3}$, Zahid Mushtaq ${ }^{3}$, \\ Syed AA Shah ${ }^{4,5}$ \\ ${ }^{1}$ Department of Chemistry, Government College University, Lahore-54000, ${ }^{2}$ Department of Pharmacy, The Islamia University of \\ Bahawalpur, Bahawalpur-63100, ${ }^{3}$ Department of Biochemistry, University of Agriculture, Faisalabad-38040, Pakistan, ${ }^{4}$ Faculty \\ of Pharmacy, ${ }^{5}$ Atta-ur-Rahman Institute for Natural Products Discovery (AuRIns), Level 9, FF3, Universiti Teknologi MARA, \\ Puncak Alam Campus, 42300 Bandar Puncak Alam, Selangor Darul Ehsan, Malaysia
}

*For correspondence: Email: atrabbasi@yahoo.com; abbasi@gcu.edu.pk; Tel: (+92)-42-111000010

\begin{abstract}
Purpose: To evaluate the antibacterial activity and cytotoxicity of a series of molecules with amalgamation of furoyl, piperazine and amide moieties.

Methods: New derivatives, namely 3-[4-(2-furoyl)-1-piperazinyl]-N-(substituted) propanamides, were synthesized and evaluated for their antibacterial activity and toxicity to mammalian cells. The synthesis was initiated by treating different aryl/aralkyl amines (1a-u) with 3-bromopropionyl chloride (2) to obtain the solid electrophiles 3a-u, which were collected by filtration. Thereafter, the different $\mathrm{N}$-aryl/aralkyl-3bromopropionamides (3a-u) and 2-furoyl-1-piperazine (4) at equimolar ratios were allowed to react in acetonitrile and in the presence of a base, $\mathrm{K}_{2} \mathrm{CO}_{3}$, to form the target compounds, 5a-u. Structural elucidation was carried out using El-MS (electron impact mass spectrometry), IR (infrared) and $1 \mathrm{H}-\mathrm{NMR}$ (proton nuclear magnetic resonance). The antibacterial activity of the synthesized compounds was evaluated against various bacterial strains. Furthermore, hemolysis was determined to assess cytotoxicity using bovine red blood cells.

Results: Molecules $\mathbf{5 g}, \mathbf{5 a}, \mathbf{5 p}, \mathbf{5 g}$ and $\mathbf{5 i}$ were found to be potent agents against $S$. aureus, S. typhi, $P$. aeruginosa, E. coli and B. subtilis with respective minimum inhibitory concentration (MIC) values of 8.34 $\pm 0.55,8.37 \pm 0.12,8.65 \pm 0.57,8.97 \pm 0.12$ and $9.24 \pm 0.50 \mu \mathrm{M}$, compared to $7.80 \pm 0.19,7.45 \pm 0.58$, $7.14 \pm 0.58,7.16 \pm 0.58$ and $7.29 \pm 0.90 \mu \mathrm{M}$ for the reference standard, ciprofloxacin. The most active compounds, 5a, $\mathbf{5 g}$, 5i and $\mathbf{5 p}$, showed a hemolysis of $15.48,8.03,5.52$ and $4.35 \%$, respectively.

Conclusion: The synthesized compounds exhibit good antibacterial activity. The hemolysis data indicate that these compounds have a low toxicity level. However, in vivo studies are required to ascertain their potentials as new drug candidates.
\end{abstract}

Keywords: 4-(2-Furoyl)-1-piperazine, ${ }^{1} \mathrm{H}-\mathrm{NMR}$, EI-MS, Antimicrobial activity, Hemolytic activity

This is an Open Access article that uses a funding model which does not charge readers or their institutions for access and distributed under the terms of the Creative Commons Attribution License (http://creativecommons.org/licenses/by/4.0) and the Budapest Open Access Initiative (http://www.budapestopenaccessinitiative.org/read), which permit unrestricted use, distribution, and reproduction in any medium, provided the original work is properly credited.

Tropical Journal of Pharmaceutical Research is indexed by Science Citation Index (SciSearch), Scopus, International Pharmaceutical Abstract, Chemical Abstracts, Embase, Index Copernicus, EBSCO, African Index Medicus, JournalSeek, Journal Citation Reports/Science Edition, Directory of Open Access Journals (DOAJ), African Journal Online, Bioline International, Open-J-Gate and Pharmacy Abstracts 


\section{INTRODUCTION}

There are wide possibilities for the utilization of furan derivatives as biologically active compounds. Piperazine derivatives are known to possess the properties of triple reuptake inhibitors [1], norovirus inhibitors [2], cannabinoid CB1 receptor agonists [3], antagonists for the melanocortin-4 receptor [4], antimicrobials [5], enzyme inhibitors [6], etc. This moiety has also found applications in the field of engineering [7] and polymers [8]. The amides are known to possess valuable biological activities [9-11].

These valuable biological activities of furan and amide moieties prompted us to introduce some new molecules with the amalgamation of these moieties and to evaluate their potential as antibacterial agents.

\section{EXPERIMENTAL}

\section{General}

Chemicals and solvents were from Sigma Aldrich and Alfa Aesar and purchased through a local supplier. All solvents were further used without purification. A Griffin and George melting point apparatus was utilized to read the melting points of synthesized compounds, and melting points were uncorrected. Thin layer chromatography (TLC) run on silica-coated aluminum plates helped to confirm product formation. The mobile phase was a mixture of ethyl acetate and $n$ hexane and spots were visualized under a UV lamp at $254 \mathrm{~nm}$. A Jasco-320-A spectrometer was used to record IR peaks by the $\mathrm{KBr}$ pellet method. Bruker spectrometers were used to record ${ }^{1} \mathrm{H}-\mathrm{NMR}$ signals at $500 \mathrm{MHz}$ in $\mathrm{CHCl}_{3}-d_{1}$, and a JMS-HX-110 spectrometer recorded EIMS signals.

\section{General procedure for synthesis of $\mathrm{N}$ - aryl/aralkyl-3-bromopropanamides (3a-u)}

A calculated amount of substituted aryl/aralkyl/alkyl amines (1a-u; $15.0 \mathrm{mmol}$ ) was placed in a $250-\mathrm{mL}$ iodine flask containing distilled water $(15.0 \mathrm{~mL})$ and $10 \% \mathrm{Na}_{2} \mathrm{CO}_{3}$. The $\mathrm{pH}$ was adjusted to 9-10. 3-Bromopropionyl chloride $(2 ; 15.0 \mathrm{mmol})$ was then added dropwise to the reaction mixture for $2-5 \mathrm{~min}$. A solid precipitate appeared on vigorous shaking. TLC was used to monitor the reaction progress. The products, $\mathrm{N}$-(aryl/aralkyl/alkyl)-3bromopropanamides (3a-u), were collected by filtration and washed and dried.
General procedure for synthesis of 3-[4-(2furoyl)-1-piperazinyl]- $\mathrm{N}$-(aryl/aralkyl/alkyl) propanamides $(5 \mathrm{a}-\mathrm{u})$

4-(2-Furoyl)-1-piperazine $(4 ; 0.1 \mathrm{~g}, 4.5 \mathrm{mmol})$ and solid $\mathrm{K}_{2} \mathrm{CO}_{3}(13.5 \mathrm{mmol})$ were dissolved in acetonitrile $(18 \mathrm{~mL})$ in a round-bottom flask (100 $\mathrm{mL}$ ). After refluxing for $0.5 \mathrm{~h}$, the electrophiles $N$ aryl/aralkyl/alkyl-3-bromopropanamides (3a-u; $4.5 \mathrm{mmol}$ ) were added and refluxing was continued for 3-4 $\mathrm{h}$. The reaction was monitored by TLC. The final compounds were acquired after the addition of excess distilled water, and the precipitate was filtered out, washed and dried.

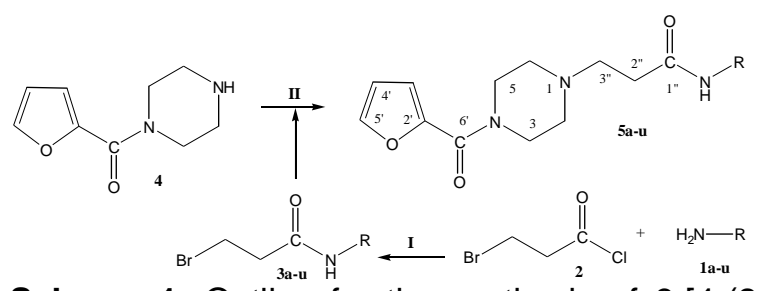

Scheme 1: Outline for the synthesis of 3-[4-(2furoyl)-1-piperazinyl]- $N$-(substituted)

propanamides $(5 a-u)$. For $-R$ group, see structures of derivatives in Figure 1.

\section{Reagents and conditions}

(I) $10 \%$ sodium carbonate, $\mathrm{pH}=9-10$, stir for 3 - $4 \mathrm{~h}$, room temperature; (II) acetonitrile, potassium carbonate, reflux for $0.5 \mathrm{~h}$; and (III) reflux for $3-4 \mathrm{~h}$.

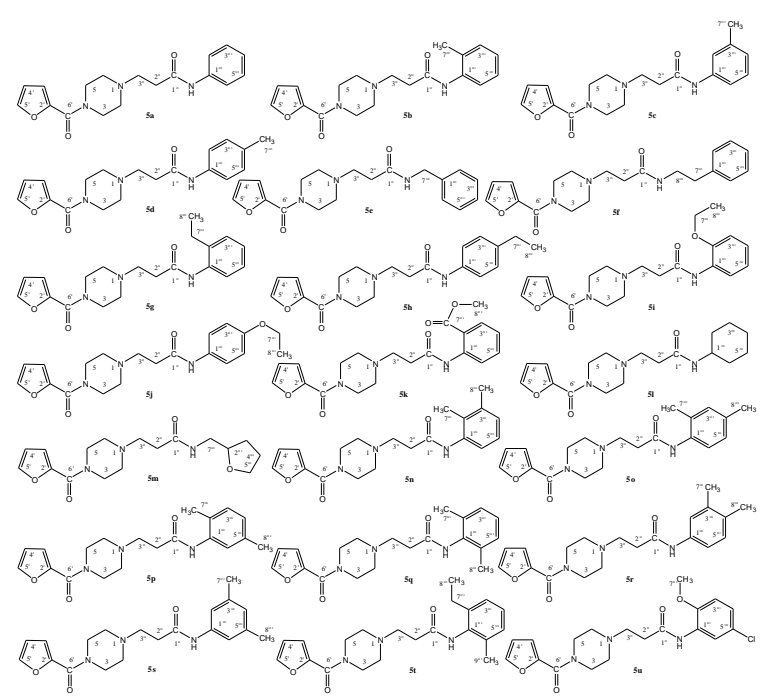

Figure 1: Structures of different derivatives

\section{Antibacterial activity assay}

The broth microdilution method was employed to evaluate the antibacterial activity of the synthesized compounds. The synthesized compounds at different concentrations were 
mixed with selected bacterial strains, and the change in absorbance before and after incubation was noted $[12,13]$.

\section{Evaluation of hemolytic activity}

Hemolytic activity was studied using a previously reported method [14,15]. A blood sample was collected from a cow, and red blood cells (RBCs) were isolated for use. Triton X-100 and phosphate buffer saline (PBS) were employed as the positive and negative controls, respectively.

\section{Statistical analysis}

Microsoft Excel 2010 was utilized for statistical analysis of results which were expressed as mean $\pm \operatorname{SEM}(n=3)$.

\section{RESULTS}

The target molecules, 3-[4-(2-furoyl)-1piperazinyl]- $N$-(aryl/aralkyl/alkyl)propanamides (5a-u), were synthesized as outlined in Scheme1 and structures of different derivatives are also shown in Figure 1.

\section{Spectral characteristics of synthesized molecules $(5 a-u)$}

\section{3-[4-(2-Furoyl)-1-piperazinyl]-N- phenylpropanamide (5a)}

Gray-brown amorphous solid; yield: 90 \%; m.p.: 105-107 ${ }^{\circ} \mathrm{C}$; Mol. F.: $\mathrm{C}_{18} \mathrm{H}_{21} \mathrm{~N}_{3} \mathrm{O}_{3}$; Mol. Mass.: 327; IR ( $\left.\mathrm{KBr}, \mathrm{cm}^{-1}\right)$ v: $3406(\mathrm{~N}-\mathrm{H}), 3086$ (Ar C-H), 2882 ( R C-H), 1657 (C=O), 1582 (Ar C=C), 1197 (C-O-C), 1110 (C-N-C); ${ }^{1} \mathrm{H}-\mathrm{NMR}(500 \mathrm{MHz}$, $\mathrm{CDCl}_{3}, \delta$ in ppm): 7.48 (distorted d, $J=1.5 \mathrm{~Hz}$, $\left.1 \mathrm{H}, \mathrm{H}-5^{\prime}\right)$, 7.33-7.27 (m, 5H, H-2'" to H-6"'), 7.04 (d, $J=3.5 \mathrm{~Hz}, 1 \mathrm{H}, \mathrm{H}-3$ ), 6.49 (dd, $J=1.7,3.4$ $\mathrm{Hz}, 1 \mathrm{H}, \mathrm{H}-4$ '), 3.94 (br.s, $\left.4 \mathrm{H}, \mathrm{CH}_{2}-3, \mathrm{CH}_{2}-5\right), 2.77$ (t, $\left.\mathrm{J}=6.2 \mathrm{~Hz}, 2 \mathrm{H}, \mathrm{CH}_{2}-2 "\right), 2.65$ (t, $\mathrm{J}=6.2 \mathrm{~Hz}$, $\left.2 \mathrm{H}, \mathrm{CH}_{2}-3 "\right), 2.63\left(\mathrm{~m}, 4 \mathrm{H}, \mathrm{CH}_{2}-2, \mathrm{CH}_{2}-4\right)$; EIMS $(m / z): \quad 327 \quad[\mathrm{M}]^{+}, \quad 216 \quad\left[\mathrm{C}_{13} \mathrm{H}_{16} \mathrm{~N}_{2} \mathrm{O}\right]^{*+}, \quad 203$ $\left[\mathrm{C}_{12} \mathrm{H}_{15} \mathrm{~N}_{2} \mathrm{O}\right]^{+}, 193\left[\mathrm{C}_{10} \mathrm{H}_{13} \mathrm{~N}_{2} \mathrm{O}_{2}\right]^{+}, 147\left[\mathrm{C}_{9} \mathrm{H}_{9} \mathrm{NO}\right]^{+}$, $138\left[\mathrm{C}_{7} \mathrm{H}_{8} \mathrm{NO}_{2}\right]^{+}, 119\left[\mathrm{C}_{7} \mathrm{H}_{5} \mathrm{NO}\right]^{+}, 95\left[\mathrm{C}_{5} \mathrm{H}_{3} \mathrm{O}_{2}\right]^{+}$, $93\left[\mathrm{C}_{6} \mathrm{H}_{7} \mathrm{~N}\right]^{*+}$.

\section{3-[4-(2-Furoyl)-1-piperazinyl]-N- methylphenyl) propanamide (5b)}

Light brown-pink amorphous solid; yield: $91 \%$; m.p.: $120-122{ }^{\circ} \mathrm{C}$; Mol. F.: $\mathrm{C}_{19} \mathrm{H}_{23} \mathrm{~N}_{3} \mathrm{O}_{3}$; Mol. Mass: 341 ; IR $\left(\mathrm{KBr}, \mathrm{cm}^{-1}\right)$ v: $3411(\mathrm{~N}-\mathrm{H}), 3069$ (Ar C-H), $2877(\mathrm{R} \mathrm{C}-\mathrm{H}), 1657(\mathrm{C}=\mathrm{O}), 1576(\mathrm{Ar}$ $\mathrm{C}=\mathrm{C}), 1204$ (C-O-C), 1114 (C-N-C); ${ }^{1} \mathrm{H}-\mathrm{NMR}$ $\left(500 \mathrm{MHz}, \mathrm{CDCl}_{3}, \delta\right.$ in ppm): 7.49 (distorted d, $J$ $\left.=1.0 \mathrm{~Hz}, 1 \mathrm{H}, \mathrm{H}-5^{\prime}\right), 7.39(\mathrm{~d}, J=7.6 \mathrm{~Hz}, 1 \mathrm{H}, \mathrm{H}-$ 6'"), 7.24 (d, $\left.J=7.6 \mathrm{~Hz}, 1 \mathrm{H}, \mathrm{H}-3{ }^{\prime \prime \prime}\right), 7.14$ (br.t, $J=$
$7.6 \mathrm{~Hz}, 1 \mathrm{H}, \mathrm{H}-5$ '"), 7.11 (dt, $J=6.6,1.0 \mathrm{~Hz}, 1 \mathrm{H}$, H-4"'), 7.05 (d, J = $\left.3.5 \mathrm{~Hz}, 1 \mathrm{H}, \mathrm{H}-3^{\prime}\right), 6.05$ (dd, $J$ $=1.7,3.4 \mathrm{~Hz}, 1 \mathrm{H}, \mathrm{H}-4^{\prime}$ ), 3.91 (br.s, $4 \mathrm{H}, \mathrm{CH}_{2}-3$, $\left.\mathrm{CH}_{2}-5\right), 2.78\left(\mathrm{t}, \mathrm{J}=6.2 \mathrm{~Hz}, 2 \mathrm{H}, \mathrm{CH}_{2}-2 "\right), 2.67(\mathrm{~m}$, $\left.4 \mathrm{H}, \mathrm{CH}_{2}-2, \mathrm{CH}_{2}-4\right), 2.56$ (t, $J=6.2 \mathrm{~Hz}, 2 \mathrm{H}, \mathrm{CH}_{2}-$ 3") 2.25 (s, 3H, $\left.\mathrm{CH}_{3}-7^{\prime \prime \prime}\right)$; EIMS (m/z): $341[\mathrm{M}]^{+}$, $230 \quad\left[\mathrm{C}_{14} \mathrm{H}_{18} \mathrm{~N}_{2} \mathrm{O}\right]^{+}, \quad 217 \quad\left[\mathrm{C}_{13} \mathrm{H}_{17} \mathrm{~N}_{2} \mathrm{O}\right]^{+}, 193$ $\left[\mathrm{C}_{10} \mathrm{H}_{13} \mathrm{~N}_{2} \mathrm{O}_{2}\right]^{+}, 161\left[\mathrm{C}_{10} \mathrm{H}_{11} \mathrm{NO}^{+}, 138\left[\mathrm{C}_{7} \mathrm{H}_{8} \mathrm{NO}_{2}\right]^{+}\right.$, $133\left[\mathrm{C}_{8} \mathrm{H}_{7} \mathrm{NO}\right]^{++}, 107\left[\mathrm{C}_{7} \mathrm{H}_{9} \mathrm{~N}\right]^{++}, 95\left[\mathrm{C}_{5} \mathrm{H}_{3} \mathrm{O}_{2}\right]^{+}$.

\section{3-[4-(2-Furoyl)-1-piperazinyl]-N-(3- methylphenyl) propanamide (5c)}

Grey-white crystalline solid; yield: 92 \%; m.p: 116-118 ${ }^{\circ} \mathrm{C}$; Mol. F.: $\mathrm{C}_{19} \mathrm{H}_{23} \mathrm{~N}_{3} \mathrm{O}_{3}$; Mol. Mass: 341; IR ( $\left.\mathrm{KBr}, \mathrm{cm}^{-1}\right)$ v: $3405(\mathrm{~N}-\mathrm{H}), 3083(\mathrm{Ar} \mathrm{C}-\mathrm{H})$, $2882(\mathrm{R} \mathrm{C}-\mathrm{H}), 1656(\mathrm{C}=\mathrm{O}), 1575(\mathrm{Ar} \mathrm{C}=\mathrm{C}), 1209$ (C-O-C), 1118 (C-N-C); ${ }^{1} \mathrm{H}-\mathrm{NMR} \quad(500 \mathrm{MHz}$, $\mathrm{CDCl}_{3}, \delta$ in ppm): 7.49 (distorted d, $J=1.0 \mathrm{~Hz}$, $\left.1 \mathrm{H}, \mathrm{H}-5^{\prime}\right), 7.36$ (br.s, $\left.1 \mathrm{H}, \mathrm{H}-2^{\prime \prime \prime}\right), 7.31$ (br.d, $J=$ $\left.8.0 \mathrm{~Hz}, 1 \mathrm{H}, \mathrm{H}-6^{\prime \prime \prime}\right), 7.17$ (br.t, $J=8.0 \mathrm{~Hz}, 1 \mathrm{H}, \mathrm{H}-$ 5"'), 7.05 (d, $\left.J=3.5 \mathrm{~Hz}, 1 \mathrm{H}, \mathrm{H}-3^{\prime}\right), 6.89$ (br.d, $J=$ $7.2 \mathrm{~Hz}, 1 \mathrm{H}, \mathrm{H}-4{ }^{\prime \prime \prime)}, 6.05$ (dd, $J=1.7,3.4 \mathrm{~Hz}, 1 \mathrm{H}$, H-4'), 3.91 (br.s, $4 \mathrm{H}, \mathrm{CH}_{2}-3, \mathrm{CH}_{2}-5$ ), 2.78 (t, $J=$ $\left.6.2 \mathrm{~Hz}, 2 \mathrm{H}, \mathrm{CH}_{2}-2 "\right), 2.67$ (m, 4H, $\left.\mathrm{CH}_{2}-2, \mathrm{CH}_{2}-4\right)$, $2.56\left(\mathrm{t}, \mathrm{J}=6.2 \mathrm{~Hz}, 2 \mathrm{H}, \mathrm{CH}_{2}-3 "\right), 2.31(3 \mathrm{H}, \mathrm{s}$, $\left.\mathrm{CH}_{3}-7^{\prime \prime \prime}\right) ; \quad \operatorname{EIMS}(\mathrm{m} / \mathrm{z}): \quad 341 \quad\left[\mathrm{M}^{+}, \quad 230\right.$ $\left[\mathrm{C}_{14} \mathrm{H}_{18} \mathrm{~N}_{2} \mathrm{O}\right]^{+}, \quad 217 \quad\left[\mathrm{C}_{13} \mathrm{H}_{17} \mathrm{~N}_{2} \mathrm{O}\right]^{+}, \quad 193$ $\left[\mathrm{C}_{10} \mathrm{H}_{13} \mathrm{~N}_{2} \mathrm{O}_{2}\right]^{+}, 161\left[\mathrm{C}_{10} \mathrm{H}_{11} \mathrm{NO}^{+}, 138\left[\mathrm{C}_{7} \mathrm{H}_{8} \mathrm{NO}_{2}\right]^{+}\right.$, $133\left[\mathrm{C}_{8} \mathrm{H}_{7} \mathrm{NO}\right]^{++}, 107\left[\mathrm{C}_{7} \mathrm{H}_{9} \mathrm{~N}\right]^{+}, 95\left[\mathrm{C}_{5} \mathrm{H}_{3} \mathrm{O}_{2}\right]^{+}$.

\section{3-[4-(2-Furoyl)-1-piperazinyl]-N-(4- methylphenyl) propanamide (5d)}

White crystalline solid; yield: $94 \%$; m.p: 122-124 ${ }^{\circ} \mathrm{C}$; Mol. F.: $\mathrm{C}_{19} \mathrm{H}_{23} \mathrm{~N}_{3} \mathrm{O}_{3}$; Mol. Mass: 341 ; IR $(\mathrm{KBr}$, $\left.\mathrm{cm}^{-1}\right)$ v: $3415(\mathrm{~N}-\mathrm{H}), 3072(\mathrm{Ar} \mathrm{C}-\mathrm{H}), 2874(\mathrm{R} \mathrm{C}-$ $\mathrm{H}), 1651(\mathrm{C}=\mathrm{O}), 1586(\mathrm{Ar} \mathrm{C}=\mathrm{C}), 1209$ (C-O-C), 1108 (C-N-C); ${ }^{1} \mathrm{H}-\mathrm{NMR}\left(500 \mathrm{MHz}, \mathrm{CDCl}_{3}, \delta\right.$ in ppm): 7.49 (distorted d, $J=1.0 \mathrm{~Hz}, 1 \mathrm{H}, \mathrm{H}-5^{\prime}$ ), 7.40 (d, $J=8.3 \mathrm{~Hz}, 2 \mathrm{H}, \mathrm{H}-2$ '", $\left.\mathrm{H}-6 \mathrm{C}^{\prime \prime}\right), 7.12$ (d, $J=$ $\left.8.2 \mathrm{~Hz}, 2 \mathrm{H}, \mathrm{H}-3^{\prime \prime \prime}, \mathrm{H}-5^{\prime \prime \prime}\right), 7.05$ (d, $J=3.5 \mathrm{~Hz}, 1 \mathrm{H}$, $\left.\mathrm{H}^{\prime} 3^{\prime}\right), 6.05\left(\mathrm{dd}, J=1.7,3.4 \mathrm{~Hz}, 1 \mathrm{H}, \mathrm{H}-4^{\prime}\right), 3.91$ (br.s, $4 \mathrm{H}, \mathrm{CH}_{2}-3, \mathrm{CH}_{2}-5$ ), 2.78 (t, $J=6.2 \mathrm{~Hz}, 2 \mathrm{H}$, $\left.\mathrm{CH}_{2}-2 "\right), 2.67\left(\mathrm{~m}, 4 \mathrm{H}, \mathrm{CH}_{2}-2, \mathrm{CH}_{2}-4\right), 2.56$ (t, $J=$ $\left.6.2 \mathrm{~Hz}, 2 \mathrm{H}, \mathrm{CH}_{2}-3^{\prime \prime}\right), 2.31$ (s, 3H, $\left.\mathrm{CH}_{3}-7^{\prime \prime \prime}\right)$; EIMS $(\mathrm{m} / \mathrm{z}): \quad 341 \quad[\mathrm{M}]^{+}, \quad 230\left[\mathrm{C}_{14} \mathrm{H}_{18} \mathrm{~N}_{2} \mathrm{O}\right]^{+}, \quad 217$ $\left[\begin{array}{llll}\left.\mathrm{C}_{13} \mathrm{H}_{17} \mathrm{~N}_{2} \mathrm{O}\right]^{+}, & 193 & {\left[\mathrm{C}_{10} \mathrm{H}_{13} \mathrm{~N}_{2} \mathrm{O}_{2}\right]^{+},} & 161\end{array}\right.$ $\left[\mathrm{C}_{10} \mathrm{H}_{11} \mathrm{NO}\right]^{+}, 138\left[\mathrm{C}_{7} \mathrm{H}_{8} \mathrm{NO}_{2}\right]^{+}, 133\left[\mathrm{C}_{8} \mathrm{H}_{7} \mathrm{NO}\right]^{+}$, $107\left[\mathrm{C}_{7} \mathrm{H}_{9} \mathrm{~N}\right]^{++}, 95\left[\mathrm{C}_{5} \mathrm{H}_{3} \mathrm{O}_{2}\right]^{+}$.

\section{3-[4-(2-Furoyl)-1-piperazinyl]-N- benzylpropanamide (5e)}

Light-brown, sticky amorphous solid; yield: $90 \%$; Mol. F.: $\mathrm{C}_{19} \mathrm{H}_{23} \mathrm{~N}_{3} \mathrm{O}_{3}$; Mol. Mass: 341; IR ( $\mathrm{KBr}$, $\left.\mathrm{cm}^{-1}\right)$ v: $3410(\mathrm{~N}-\mathrm{H}), 3092(\mathrm{Ar} \mathrm{C}-\mathrm{H}), 2891$ (R C$\mathrm{H}), 1661(\mathrm{C}=\mathrm{O}), 1588(\mathrm{Ar} \mathrm{C}=\mathrm{C}), 1195(\mathrm{C}-\mathrm{O}-\mathrm{C})$, 1112 (C-N-C); ${ }^{1} \mathrm{H}-\mathrm{NMR}\left(500 \mathrm{MHz}, \mathrm{CDCl}_{3}, \delta\right.$ in ppm): 7.49 (distorted d, $\left.J=1.0 \mathrm{~Hz}, 1 \mathrm{H}, \mathrm{H}-5^{\prime}\right)$, 
7.27-7.19 (m, 5H, H-2"' to H-6"'), 7.05 (d, J = 3.5 $\left.\mathrm{Hz}, 1 \mathrm{H}, \mathrm{H}-3^{\prime}\right), 6.05$ (dd, $J=1.7,3.4 \mathrm{~Hz}, 1 \mathrm{H}, \mathrm{H}-4$ '), 4.41 (s, 2H, CH${ }_{2}-7^{\prime \prime \prime}$ ); 3.91 (br.s, $4 \mathrm{H}, \mathrm{CH}_{2}-3, \mathrm{CH}_{2-}$ 5), 2.78 (t, J = 6.2 Hz, 2H, $\left.\mathrm{CH}_{2}-2 "\right), 2.67(\mathrm{~m}, 4 \mathrm{H}$, $\left.\mathrm{CH}_{2}-2, \mathrm{CH}_{2}-4\right), 2.56$ (t, $\left.\mathrm{J}=6.2 \mathrm{~Hz}, 2 \mathrm{H}, \mathrm{CH}_{2}-3 "\right)$; EIMS $(\mathrm{m} / \mathrm{z}): 341[\mathrm{M}]^{+}, 230\left[\mathrm{C}_{14} \mathrm{H}_{18} \mathrm{~N}_{2} \mathrm{O}\right]^{++}, 217$ $\left[\mathrm{C}_{13} \mathrm{H}_{17} \mathrm{~N}_{2} \mathrm{O}\right]^{+}, \quad 193 \quad\left[\mathrm{C}_{10} \mathrm{H}_{13} \mathrm{~N}_{2} \mathrm{O}_{2}\right]^{+}, \quad 161$ $\left[\mathrm{C}_{10} \mathrm{H}_{11} \mathrm{NO}\right]^{+}, 138\left[\mathrm{C}_{7} \mathrm{H}_{8} \mathrm{NO}_{2}\right]^{+}, 133\left[\mathrm{C}_{8} \mathrm{H}_{7} \mathrm{NO}\right]^{++}$, $107\left[\mathrm{C}_{7} \mathrm{H}_{9} \mathrm{~N}\right]^{++}, 95\left[\mathrm{C}_{5} \mathrm{H}_{3} \mathrm{O}_{2}\right]^{+}$.

\section{3-[4-(2-Furoyl)-1-piperazinyl]-N-(2- phenylethyl) propanamide (5f)}

Brown amorphous solid; yield: $90 \%$; M.P: $107-$ $109{ }^{\circ} \mathrm{C}$; Mol. F.: $\mathrm{C}_{20} \mathrm{H}_{25} \mathrm{~N}_{3} \mathrm{O}_{3}$; Mol. Mass: 355; IR $\left(\mathrm{KBr}, \mathrm{cm}^{-1}\right)$ v: $3410(\mathrm{~N}-\mathrm{H}), 3090(\mathrm{Ar} \mathrm{C}-\mathrm{H}), 2880$ (R C-H), 1659 (C=O), 1581 ( $\mathrm{Ar} \mathrm{C}=\mathrm{C}), 1197$ (C-OC), 1115 (C-N-C); ${ }^{1} \mathrm{H}-\mathrm{NMR}\left(500 \mathrm{MHz}, \mathrm{CDCl}_{3}, \delta\right.$ in ppm): 7.49 (distorted $\mathrm{d}, J=1.0 \mathrm{~Hz}, 1 \mathrm{H}, \mathrm{H}-5^{\prime}$ ), 7.12-7.10 (m, 5H, H-2"' to H-6"'), 7.05 (d, J = 3.5 $\left.\mathrm{Hz}, 1 \mathrm{H}, \mathrm{H}-3^{\prime}\right), 6.05$ (dd, $J=1.7,3.4 \mathrm{~Hz}, 1 \mathrm{H}, \mathrm{H}-4^{\prime}$ ), 3.91 (br.s, $4 \mathrm{H}, \mathrm{CH}_{2}-3, \mathrm{CH}_{2}-5$ ), 3.37 (t, J = 6.2 Hz, $\left.2 \mathrm{H}, \mathrm{CH}_{2}-8^{\prime \prime \prime}\right), 2.78$ (t, J = 6.2 Hz, 2H, $\left.\mathrm{CH}_{2}-2 "\right)$, $2.67\left(\mathrm{~m}, 4 \mathrm{H}, \mathrm{CH}_{2}-2, \mathrm{CH}_{2}-4\right), 2.56(\mathrm{t}, J=6.2 \mathrm{~Hz}$, $\left.2 \mathrm{H}, \mathrm{CH}_{2}-3^{\prime \prime}\right), 2.13$ (t, $\left.\mathrm{J}=6.2 \mathrm{~Hz}, 2 \mathrm{H}, \mathrm{CH}_{2}-7^{\prime \prime \prime}\right)$; EIMS $(\mathrm{m} / \mathrm{z}): 355[\mathrm{M}]^{+}, 244\left[\mathrm{C}_{15} \mathrm{H}_{20} \mathrm{~N}_{2} \mathrm{O}\right]^{++}, 231$ $\left[\mathrm{C}_{14} \mathrm{H}_{19} \mathrm{~N}_{2} \mathrm{O}\right]^{+}, \quad 193 \quad\left[\mathrm{C}_{10} \mathrm{H}_{13} \mathrm{~N}_{2} \mathrm{O}_{2}\right]^{+}, \quad 175$ $\left[\mathrm{C}_{11} \mathrm{H}_{13} \mathrm{NO}\right]^{+}, 147\left[\mathrm{C}_{9} \mathrm{H}_{9} \mathrm{NO}\right]^{++}, 138\left[\mathrm{C}_{7} \mathrm{H}_{8} \mathrm{NO}_{2}\right]^{+}$, $121\left[\mathrm{C}_{8} \mathrm{H}_{11} \mathrm{~N}\right]^{\cdot+}, 95\left[\mathrm{C}_{5} \mathrm{H}_{3} \mathrm{O}_{2}\right]^{+}$.

\section{3-[4-(2-Furoyl)-1-piperazinyl]-N-(2- ethylphenyl) propanamide (5g)}

Light-brown amorphous solid; yield: $90 \%$; m.p: 95-97 ${ }^{\circ} \mathrm{C}$; Mol. F.: $\mathrm{C}_{20} \mathrm{H}_{25} \mathrm{~N}_{3} \mathrm{O}_{3}$; Mol. Mass: 355; IR $\left(\mathrm{KBr}, \mathrm{cm}^{-1}\right)$ v. $3407(\mathrm{~N}-\mathrm{H}), 3087(\mathrm{Ar} \mathrm{C}-\mathrm{H})$, 2883 (R C-H), 1651 (C=O), 1580 ( $\mathrm{Ar} \mathrm{C}=\mathrm{C}), 1200$ (C-O-C), 1115 (C-N-C); ${ }^{1} \mathrm{H}-\mathrm{NMR} \quad(500 \mathrm{MHz}$, $\mathrm{CDCl}_{3}, \delta$ in ppm): 7.49 (distorted $\mathrm{d}, J=1.0 \mathrm{~Hz}$, $\left.1 \mathrm{H}, \mathrm{H}-5^{\prime}\right), 7.18$ (dd, $J=9.0,3.0 \mathrm{~Hz}, 1 \mathrm{H}, \mathrm{H}-6^{\prime \prime \prime}$ ), 7.14 (dt, $\left.J=8.0,2.0 \mathrm{~Hz}, 1 \mathrm{H}, \mathrm{H}-5^{\prime \prime \prime}\right), 7.06$ (dt, $J=$ 8.5, 3.0 Hz, $1 \mathrm{H}, \mathrm{H}-4$ "'), 7.05 (d, J=3.5 Hz, $1 \mathrm{H}$, H-3'), 6.95 (dd, J = 8.0, 3.0 Hz, 1H, H-3"'), 6.05 (dd, $J=1.7,3.4 \mathrm{~Hz}, 1 \mathrm{H}, \mathrm{H}-4$ '), 3.91 (br.s, 4H, $\left.\mathrm{CH}_{2}-3, \mathrm{CH}_{2}-5\right)$, 2.78 (t, J = 6.2 Hz, 2H, $\left.\mathrm{CH}_{2}-2 "\right)$, $2.67\left(\mathrm{~m}, 4 \mathrm{H}, \mathrm{CH}_{2}-2, \mathrm{CH}_{2}-4\right), 2.56(\mathrm{t}, \mathrm{J}=6.2 \mathrm{~Hz}$, $\left.2 \mathrm{H}, \mathrm{CH}_{2}-3^{\prime \prime}\right), 2.48$ (q, $\left.J=7.5 \mathrm{~Hz}, 2 \mathrm{H}, \mathrm{CH}_{2}-7^{\prime \prime \prime}\right)$, 1.01 (t, $\left.J=7.5 \mathrm{~Hz}, 3 \mathrm{H}, \mathrm{CH}_{3}-8^{\prime \prime \prime}\right)$; $\operatorname{EIMS}(\mathrm{m} / \mathrm{z}): 355$ $[\mathrm{M}]^{+}, 244\left[\mathrm{C}_{15} \mathrm{H}_{20} \mathrm{~N}_{2} \mathrm{O}\right]^{\circ+}, 231\left[\mathrm{C}_{14} \mathrm{H}_{19} \mathrm{~N}_{2} \mathrm{O}\right]^{+}, 193$ $\left[\mathrm{C}_{10} \mathrm{H}_{13} \mathrm{~N}_{2} \mathrm{O}_{2}\right]^{+}, 175\left[\mathrm{C}_{11} \mathrm{H}_{13} \mathrm{NO}\right]^{+}, 147\left[\mathrm{C}_{9} \mathrm{H}_{9} \mathrm{NO}\right]^{++}$, $138\left[\mathrm{C}_{7} \mathrm{H}_{8} \mathrm{NO}_{2}\right]^{+}, 121\left[\mathrm{C}_{8} \mathrm{H}_{11} \mathrm{~N}\right]^{\cdot+}, 95\left[\mathrm{C}_{5} \mathrm{H}_{3} \mathrm{O}_{2}\right]^{+}$.

\section{3-[4-(2-Furoyl)-1-piperazinyl]-N-(4- ethylphenyl) propanamide (5h)}

Brown amorphous solid; yield: 90 \%; m.p: 106$108{ }^{\circ} \mathrm{C}$; Mol. F.: $\mathrm{C}_{20} \mathrm{H}_{25} \mathrm{~N}_{3} \mathrm{O}_{3}$; Mol. Mass: 355; IR $\left(\mathrm{KBr}, \mathrm{cm}^{-1}\right)$ v: $3409(\mathrm{~N}-\mathrm{H}), 3090(\mathrm{Ar} \mathrm{C}-\mathrm{H}), 2881$ (R C-H), 1658 (C=O), 1581 ( $\mathrm{Ar} \mathrm{C}=\mathrm{C}), 1197$ (C-OC), 1118 (C-N-C); ${ }^{1} \mathrm{H}-\mathrm{NMR}\left(500 \mathrm{MHz}, \mathrm{CDCl}_{3}, \delta\right.$ in ppm): 7.49 (distorted $\mathrm{d}, \mathrm{J}=1.0 \mathrm{~Hz}, 1 \mathrm{H}, \mathrm{H}-5^{\prime}$ ), 7.06 (d, J = 8.0 Hz, 2H, H-2'", H-6"'), 7.05 (d, J = $\left.3.5 \mathrm{~Hz}, 1 \mathrm{H}, \mathrm{H}-3^{\prime}\right), 6.96$ (d, J = 8.0 Hz, 2H, H-3"', H-5"'), 6.05 (dd, J = 1.7, $3.4 \mathrm{~Hz}, 1 \mathrm{H}, \mathrm{H}-4$ '), 3.91 (br.s, 4H, $\mathrm{CH}_{2}-3, \mathrm{CH}_{2}-5$ ), 2.78 (t, J = 6.2 Hz, 2H, $\left.\mathrm{CH}_{2}-2 "\right), 2.67$ (m, 4H, $\left.\mathrm{CH}_{2}-2, \mathrm{CH}_{2}-4\right), 2.56$ (t, $\mathrm{J}=$ $\left.6.2 \mathrm{~Hz}, 2 \mathrm{H}, \mathrm{CH}_{2}-3^{\prime \prime}\right), 2.54$ (q, J = $7.5 \mathrm{~Hz}, 2 \mathrm{H}$, $\left.\mathrm{CH}_{2}-7^{\prime \prime \prime}\right), 1.15$ (t, J=7.5 Hz, 3H, $\left.\mathrm{CH}_{3}-8^{\prime \prime \prime}\right)$; EIMS $(\mathrm{m} / \mathrm{z}): 355 \quad[\mathrm{M}]^{+}, \quad 244 \quad\left[\mathrm{C}_{15} \mathrm{H}_{20} \mathrm{~N}_{2} \mathrm{O}\right]^{++}, 231$ $\left[\mathrm{C}_{14} \mathrm{H}_{19} \mathrm{~N}_{2} \mathrm{O}\right]^{+}, \quad 193 \quad\left[\mathrm{C}_{10} \mathrm{H}_{13} \mathrm{~N}_{2} \mathrm{O}_{2}\right]^{+}, \quad 175$ $\left[\mathrm{C}_{11} \mathrm{H}_{13} \mathrm{NO}\right]^{+}, 147\left[\mathrm{C}_{9} \mathrm{H}_{9} \mathrm{NO}\right]^{++}, 138\left[\mathrm{C}_{7} \mathrm{H}_{8} \mathrm{NO}_{2}\right]^{+}$, $121\left[\mathrm{C}_{8} \mathrm{H}_{11} \mathrm{~N}\right]^{++}, 95\left[\mathrm{C}_{5} \mathrm{H}_{3} \mathrm{O}_{2}\right]^{+}$.

\section{3-[4-(2-Furoyl)-1-piperazinyl]-N-(2- ethoxyphenyl) propanamide (5i)}

Light-brown amorphous solid; yield: 90\%; M.P: 137-139 ${ }^{\circ} \mathrm{C}$; Mol. F.: $\mathrm{C}_{20} \mathrm{H}_{25} \mathrm{~N}_{3} \mathrm{O}_{4}$; Mol. Mass: 371; IR (KBr, cm $\left.{ }^{-1}\right)$ v: $3409(\mathrm{~N}-\mathrm{H}), 3086(\mathrm{Ar} \mathrm{C}-\mathrm{H})$, 2881 (R C-H), 1658 (C=O), 1583 ( $\mathrm{Ar} \mathrm{C}=\mathrm{C}), 1197$ (C-O-C), 1116 (C-N-C); ${ }^{1} \mathrm{H}-\mathrm{NMR}$ (500 MHz, $\mathrm{CDCl}_{3}, \delta$ in ppm): 8.63 (br.d, $J=8.4 \mathrm{~Hz}, 1 \mathrm{H}, \mathrm{H}-$ 6"'), 8.00 (dd, J = 8.0, 1.2 Hz, 1H, H-3"'), 7.55 (dt, $J=8.8,1.2 \mathrm{~Hz}, 1 \mathrm{H}, \mathrm{H}-5$ "'), 7.49 (distorted d, $J=$ $1.0 \mathrm{~Hz}, 1 \mathrm{H}, \mathrm{H}-5$ ), 7.12 (dt, J=8.6, $1.2 \mathrm{~Hz}, 1 \mathrm{H}, \mathrm{H}-$ 4"'), 7.05 (d, J = 3.5 Hz, 1H, H-3'), 6.05 (dd, $J=$ 1.7, $3.4 \mathrm{~Hz}, 1 \mathrm{H}, \mathrm{H}-4$ ), 3.91 (br.s, $4 \mathrm{H}, \mathrm{CH}_{2}-3$, $\left.\mathrm{CH}_{2}-5\right), 3.73$ (q, J = 7.5 Hz, 2H, $\left.\mathrm{CH}_{2}-7^{\prime \prime \prime}\right), 2.78$ (t, $\left.J=6.2 \mathrm{~Hz}, 2 \mathrm{H}, \mathrm{CH}_{2}-2^{\prime \prime}\right), 2.67\left(\mathrm{~m}, 4 \mathrm{H}, \mathrm{CH}_{2}-2\right.$, $\left.\mathrm{CH}_{2}-4\right), 2.56$ (t, J = 6.2 Hz, 2H, $\left.\mathrm{CH}_{2}-3 "\right), 1.14$ (t, J $\left.=7.5 \mathrm{~Hz}, 3 \mathrm{H}, \mathrm{CH}_{3}-8^{\prime \prime \prime}\right)$; EIMS $(\mathrm{m} / \mathrm{z}): 371\left[\mathrm{M}^{+}, 260\right.$ $\left[\mathrm{C}_{15} \mathrm{H}_{20} \mathrm{~N}_{2} \mathrm{O}_{2}\right]^{++}, \quad 247 \quad\left[\mathrm{C}_{14} \mathrm{H}_{19} \mathrm{~N}_{2} \mathrm{O}_{2}\right]^{+}, \quad 193$ $\left[\mathrm{C}_{10} \mathrm{H}_{13} \mathrm{~N}_{2} \mathrm{O}_{2}\right]^{+}, \quad 191 \quad\left[\mathrm{C}_{11} \mathrm{H}_{13} \mathrm{NO}_{2}\right]^{+}, \quad 163$ $\left[\mathrm{C}_{9} \mathrm{H}_{9} \mathrm{NO}_{2}\right]^{++}, 138\left[\mathrm{C}_{7} \mathrm{H}_{8} \mathrm{NO}_{2}\right]^{+}, 137\left[\mathrm{C}_{8} \mathrm{H}_{11} \mathrm{NO}\right]^{++}$, $95\left[\mathrm{C}_{5} \mathrm{H}_{3} \mathrm{O}_{2}\right]^{+}$.

\section{3-[4-(2-Furoyl)-1-piperazinyl]-N-(4- ethoxyphenyl) propanamide (5j)}

Tea-pink amorphous solid; yield: $90 \%$; M.P: 142$144{ }^{\circ} \mathrm{C}$; Mol. F.: $\mathrm{C}_{20} \mathrm{H}_{25} \mathrm{~N}_{3} \mathrm{O}_{4}$; Mol. Mass: 371; IR $\left(\mathrm{KBr}, \mathrm{cm}^{-1}\right)$ v: $3412(\mathrm{~N}-\mathrm{H}), 3084(\mathrm{Ar} \mathrm{C}-\mathrm{H}), 2887$ (R C-H), 1659 (C=O), 1583 (Ar C=C), 1195 (C-OC), 1112 (C-N-C); ${ }^{1} \mathrm{H}-\mathrm{NMR}\left(500 \mathrm{MHz}, \mathrm{CDCl}_{3}, \delta\right.$ in ppm): 7.49 (distorted $d, J=1.0 \mathrm{~Hz}, 1 \mathrm{H}, \mathrm{H}-5^{\prime}$ ), 7.05 (d, J = 3.5 Hz, 1H, H-3'), 6.93 (d, J=8.4 Hz, $1 \mathrm{H}, \mathrm{H}-2^{\prime \prime \prime}, \mathrm{H}-6$ "'), 6.75 (d, J = 8.0 Hz, 1H, H-3"', H-5"'), 6.05 (dd, J = 1.7, $3.4 \mathrm{~Hz}, 1 \mathrm{H}, \mathrm{H}-4$ '), 3.94 (q, $\left.J=7.5,2 \mathrm{H}, \mathrm{CH}_{2}-7^{\prime \prime \prime}\right), 3.91$ (br.s, $4 \mathrm{H}, \mathrm{CH}_{2}-3$, $\left.\mathrm{CH}_{2}-5\right), 2.78$ (t, J = 6.2 Hz, 2H, $\left.\mathrm{CH}_{2}-2 "\right), 2.67$ (m, $\left.4 \mathrm{H}, \mathrm{CH}_{2}-2, \mathrm{CH}_{2}-4\right), 2.56\left(\mathrm{t}, \mathrm{J}=6.2 \mathrm{~Hz}, 2 \mathrm{H}, \mathrm{CH}_{2}-\right.$ 3"), 1.32 (t, $\left.J=7.5,3 \mathrm{H}, \mathrm{CH}_{3}-8^{\prime \prime \prime}\right)$; $\operatorname{EIMS~}(\mathrm{m} / \mathrm{z})$ : $371[\mathrm{M}]^{+}, 260\left[\mathrm{C}_{15} \mathrm{H}_{20} \mathrm{~N}_{2} \mathrm{O}_{2}\right]^{*+}, 247\left[\mathrm{C}_{14} \mathrm{H}_{19} \mathrm{~N}_{2} \mathrm{O}_{2}\right]^{+}$, $193\left[\mathrm{C}_{10} \mathrm{H}_{13} \mathrm{~N}_{2} \mathrm{O}_{2}\right]^{+}, \quad 191 \quad\left[\mathrm{C}_{11} \mathrm{H}_{13} \mathrm{NO}_{2}\right]^{+}, 163$ $\left[\mathrm{C}_{9} \mathrm{H}_{9} \mathrm{NO}_{2}\right]^{++}, 138\left[\mathrm{C}_{7} \mathrm{H}_{8} \mathrm{NO}_{2}\right]^{+}, 137\left[\mathrm{C}_{8} \mathrm{H}_{11} \mathrm{NO}\right]^{++}$, $95\left[\mathrm{C}_{5} \mathrm{H}_{3} \mathrm{O}_{2}\right]^{+}$.

\section{3-[4-(2-Furoyl)-1-piperazinyl]-N-(2- methoxycarbonylphenyl) propanamide (5k)}


Light-brown amorphous solid; yield: 90\%; m.p: 107-109 ${ }^{\circ} \mathrm{C}$; Mol. F.: $\mathrm{C}_{20} \mathrm{H}_{23} \mathrm{~N}_{3} \mathrm{O}_{5}$; Mol. Mass: 385; IR ( $\left.\mathrm{KBr}, \mathrm{cm}^{-1}\right)$ v: $3408(\mathrm{~N}-\mathrm{H}), 3088(\mathrm{Ar} \mathrm{C}-\mathrm{H})$, $2884(\mathrm{R} \mathrm{C}-\mathrm{H}), 1655(\mathrm{C}=\mathrm{O}), 1584(\mathrm{Ar} \mathrm{C}=\mathrm{C}), 1199$ (C-O-C), 1114 (C-N-C); ${ }^{1} \mathrm{H}-\mathrm{NMR}(500 \mathrm{MHz}$, $\mathrm{CDCl}_{3}, \delta$ in ppm): 8.76 (br.d, $J=8.4 \mathrm{~Hz}, 1 \mathrm{H}, \mathrm{H}-$ 6"'), 8.12 (br.d, J = 7.7 Hz, 1H, H-3"'), 7.50 (br.t, $\left.J=7.7 \mathrm{~Hz}, 1 \mathrm{H}, \mathrm{H}-5^{\prime \prime \prime}\right), 7.49$ (distorted d, $J=1.5$ $\left.\mathrm{Hz}, 1 \mathrm{H}, \mathrm{H}-5^{\prime}\right), 7.12$ (br.t, $J=7.7 \mathrm{~Hz}, 1 \mathrm{H}, \mathrm{H}-4$ "'), $7.02(\mathrm{~d}, J=3.5 \mathrm{~Hz}, 1 \mathrm{H}, \mathrm{H}-3$ ') 6.50 (dd, $J=1.6$, $3.4 \mathrm{~Hz}, 1 \mathrm{H}, \mathrm{H}-4$ '), 3.92 (br.s, 4H, H-3, H-5), 3.90 (s, 3H, $\left.\mathrm{CH}_{3}-8^{\prime \prime \prime}\right) 2.76$ (t, $\left.J=6.1 \mathrm{~Hz}, 2 \mathrm{H}, \mathrm{H}-2 "\right)$, 2.61 (t, J = 6.2 Hz, 2H, H-3"), $2.60(\mathrm{~m}, 4 \mathrm{H}, \mathrm{H}-2$, $\mathrm{H}-4)$; $\operatorname{EIMS}(\mathrm{m} / \mathrm{z}): 385[\mathrm{M}]^{+}, 284\left[\mathrm{C}_{15} \mathrm{H}_{18} \mathrm{~N}_{2} \mathrm{O}_{3}\right]^{++}$, $261 \quad\left[\mathrm{C}_{14} \mathrm{H}_{17} \mathrm{~N}_{2} \mathrm{O}_{3}\right]^{+}, \quad 205 \quad\left[\mathrm{C}_{11} \mathrm{H}_{11} \mathrm{NO}_{3}\right]^{+}, \quad 193$ $\left[\mathrm{C}_{10} \mathrm{H}_{13} \mathrm{~N}_{2} \mathrm{O}_{2}\right]^{+}, 177\left[\mathrm{C}_{9} \mathrm{H}_{7} \mathrm{NO}_{3}\right]^{++}, 151\left[\mathrm{C}_{8} \mathrm{H}_{9} \mathrm{NO}_{2}\right]^{++}$, $138\left[\mathrm{C}_{7} \mathrm{H}_{8} \mathrm{NO}_{2}\right]^{+}, 95\left[\mathrm{C}_{5} \mathrm{H}_{3} \mathrm{O}_{2}\right]^{+}$.

\section{3-[4-(2-Furoyl)-1-piperazinyl]-N- cyclohexylpropanamide (5I)}

Dark-brown amorphous solid; yield: $94 \%$; m.p: 109-111 ${ }^{\circ} \mathrm{C}$; Mol. F.: $\mathrm{C}_{18} \mathrm{H}_{27} \mathrm{~N}_{3} \mathrm{O}_{3}$; Mol. Mass: 333; IR ( $\left.\mathrm{KBr}, \mathrm{cm}^{-1}\right)$ v: 3407 (N-H), 3079 (Ar C-H), 2884 (R C-H), 1652 (C=O), 1584 ( $\mathrm{Ar} \mathrm{C}=\mathrm{C}), 1198$ (C-O-C), 1107 (C-N-C); ${ }^{1} \mathrm{H}-\mathrm{NMR} \quad(500 \mathrm{MHz}$, $\mathrm{CDCl}_{3}, \delta$ in ppm): 7.49 (distorted d, $J=1.0 \mathrm{~Hz}$, $\left.1 \mathrm{H}, \mathrm{H}-5^{\prime}\right), 7.05$ (d, J = $\left.3.5 \mathrm{~Hz}, 1 \mathrm{H}, \mathrm{H}-3^{\prime}\right), 6.05$ (dd, $J=1.7,3.4 \mathrm{~Hz}, 1 \mathrm{H}, \mathrm{H}-4$ '), 3.91 (br.s, $4 \mathrm{H}, \mathrm{CH}_{2}-3$, $\left.\mathrm{CH}_{2}-5\right)$, 3.85-3.83 (m, 1H, H-1'"), 2.78 (t, $\mathrm{J}=6.2$ $\left.\mathrm{Hz}, 2 \mathrm{H}, \mathrm{CH}_{2}-2 "\right), 2.67\left(\mathrm{~m}, 4 \mathrm{H}, \mathrm{CH}_{2}-2, \mathrm{CH}_{2}-4\right)$, $2.56\left(\mathrm{t}, \mathrm{J}=6.2 \mathrm{~Hz}, 2 \mathrm{H}, \mathrm{CH}_{2}-3^{\prime \prime}\right), 1.86-1.14(\mathrm{~m}$, $10 \mathrm{H}, \mathrm{CH}_{2}-2$ "' to $\mathrm{CH}_{2}-6$ "'); EIMS (m/z): $333[\mathrm{M}]^{+}$, $222\left[\mathrm{C}_{17} \mathrm{H}_{18} \mathrm{~N}_{2} \mathrm{O}\right]^{++}, 209 \quad\left[\mathrm{C}_{16} \mathrm{H}_{17} \mathrm{~N}_{2} \mathrm{O}\right]^{+}, 193$ $\left[\mathrm{C}_{10} \mathrm{H}_{13} \mathrm{~N}_{2} \mathrm{O}_{2}\right]^{+}, 152\left[\mathrm{C}_{13} \mathrm{H}_{11} \mathrm{NO}\right]^{+}, 138\left[\mathrm{C}_{7} \mathrm{H}_{8} \mathrm{NO}_{2}\right]^{+}$, $125\left[\mathrm{C}_{7} \mathrm{H}_{11} \mathrm{NO}\right]^{*+}, 99\left[\mathrm{C}_{6} \mathrm{H}_{13} \mathrm{~N}\right]^{++}, 95\left[\mathrm{C}_{5} \mathrm{H}_{3} \mathrm{O}_{2}\right]^{+}$.

\section{3-[4-(2-Furoyl)-1-piperazinyl]-N- tetrahydrofuran-2-ylmethylpropanamide $(5 \mathrm{~m})$}

Light-brown amorphous solid; yield: $90 \%$ m.p: 107-109 ${ }^{\circ} \mathrm{C}$; Mol. F.: $\mathrm{C}_{17} \mathrm{H}_{25} \mathrm{~N}_{3} \mathrm{O}_{4}$; Mol. Mass: 335; IR ( $\left.\mathrm{KBr}, \mathrm{cm}^{-1}\right)$ v: $3406(\mathrm{~N}-\mathrm{H}), 3085(\mathrm{Ar} \mathrm{C}-\mathrm{H})$, 2884 (R C-H), 1655 (C=O), 1584 (Ar C=C), 1196 (C-O-C), 1112 (C-N-C); ${ }^{1} \mathrm{H}-\mathrm{NMR}(500 \mathrm{MHz}$, $\mathrm{CDCl}_{3}, \delta$ in ppm): 7.48 (distorted $\mathrm{d}, J=1.4 \mathrm{~Hz}$, $\left.1 \mathrm{H}, \mathrm{H}-5^{\prime}\right), 7.05$ (d, J = 3.4 Hz, 1H, H-3'), 6.48 (dd, $\left.J=1.7,3.4 \mathrm{~Hz}, 1 \mathrm{H}, \mathrm{H}-4^{\prime}\right), 4.05-3.98(\mathrm{~m}, 1 \mathrm{H}, \mathrm{H}-$ 2"'), 3.92 (br.s, 4H, H-3, H-5), 3.84-3.82 (m, 1H, $\mathrm{H}_{\mathrm{eq}}{ }^{-5}$ "'), 3.71-3.67 (m, 1H, $\left.\mathrm{H}_{\mathrm{ax}}-5^{\prime \prime \prime}\right), 3.21$ (dd, $J=$ 14.0, $\left.6.8 \mathrm{~Hz}, 1 \mathrm{H}, \mathrm{H}_{\mathrm{a}}-6^{\prime \prime \prime}\right), 3.11$ (dd, $J=14.8,7.2$ $\left.\mathrm{Hz}, 1 \mathrm{H}, \mathrm{H}_{\mathrm{b}}-6^{\prime \prime \prime}\right), 2.76$ (t, $\left.J=6.2 \mathrm{~Hz}, 2 \mathrm{H}, \mathrm{H}-2^{\prime \prime}\right)$, 2.65 (t, J = 6.2 Hz, 2H, H-3"), 2.62 (m, 4H, $\mathrm{CH}_{2-}$ 2, $\left.\mathrm{CH}_{2}-4\right)$, 2.03-1.95 (m, $\left.1 \mathrm{H}, \mathrm{H}_{\mathrm{eq}}-4^{\prime \prime \prime}\right), 1.90-1.84$ (m, 1H, $\left.\mathrm{H}_{\mathrm{eq}}-3^{\prime \prime \prime}\right), 1.69-1.59$ (m, 2H, $\mathrm{H}_{\mathrm{ax}}-3^{\prime \prime \prime}, \mathrm{H}_{\mathrm{ax}}$ $4^{\prime \prime \prime)}$; $\operatorname{EIMS}(\mathrm{m} / \mathrm{z})$ : $\operatorname{EIMS}(\mathrm{m} / \mathrm{z}): 335[\mathrm{M}]^{+}, 224$ $\left[\mathrm{C}_{12} \mathrm{H}_{20} \mathrm{~N}_{2} \mathrm{O}_{2}\right]^{\circ+}, \quad 211 \quad\left[\mathrm{C}_{11} \mathrm{H}_{19} \mathrm{~N}_{2} \mathrm{O}_{2}\right]^{+}, \quad 193$ $\left[\mathrm{C}_{10} \mathrm{H}_{13} \mathrm{~N}_{2} \mathrm{O}_{2}\right]^{+}, 155\left[\mathrm{C}_{8} \mathrm{H}_{13} \mathrm{NO}_{2}\right]^{+}, 138\left[\mathrm{C}_{7} \mathrm{H}_{8} \mathrm{NO}_{2}\right]^{+}$, $127\left[\mathrm{C}_{6} \mathrm{H}_{9} \mathrm{NO}_{2}\right]^{*+}, 99\left[\mathrm{C}_{5} \mathrm{H}_{9} \mathrm{NO}^{++}, 95\left[\mathrm{C}_{5} \mathrm{H}_{3} \mathrm{O}_{2}\right]^{+}\right.$.
3-[4-(2-Furoyl)-1-piperazinyl]-N-(2,3dimethylphenyl) propanamide (5n)

Light-brown amorphous solid; yield: 90 \%; m.p: 121-123 ${ }^{\circ} \mathrm{C}$; Mol. F.: $\mathrm{C}_{20} \mathrm{H}_{25} \mathrm{~N}_{3} \mathrm{O}_{3}$; Mol. Mass: 355; IR ( $\left.\mathrm{KBr}, \mathrm{cm}^{-1}\right)$ v: $3410(\mathrm{~N}-\mathrm{H}), 3075(\mathrm{Ar} \mathrm{C}-\mathrm{H})$, 2880 (R C-H), 1649 (C=O), 1580 (Ar C=C), 1205 (C-O-C), 1110 (C-N-C); ${ }^{1} \mathrm{H}-\mathrm{NMR}$ (500 MHz, $\mathrm{CDCl}_{3}, \delta$ in ppm): 7.57 (br.d, $J=7.6 \mathrm{~Hz}, 1 \mathrm{H}, \mathrm{H}-$ 6"'), 7.49 (distorted d, J=1.0 Hz, 1H, H-5'), 7.11 (br.t, $\left.J=8.0 \mathrm{~Hz}, 1 \mathrm{H}, \mathrm{H}-5^{\prime \prime \prime}\right), 7.05$ (d, $J=3.5 \mathrm{~Hz}$, $1 \mathrm{H}, \mathrm{H}-3$ '), 7.03 (br.d, J=7.6 Hz, 1H, H-4"'), 6.05 (dd, $J=1.7,3.4 \mathrm{~Hz}, 1 \mathrm{H}, \mathrm{H}-4$ '), 3.91 (br.s, 4H, $\mathrm{CH}_{2}-3, \mathrm{CH}_{2}-5$ ), 2.78 (t, J = 6.2 Hz, 2H, $\mathrm{CH}_{2}-2$ "), $2.67\left(\mathrm{~m}, 4 \mathrm{H}, \mathrm{CH}_{2}-2, \mathrm{CH}_{2}-4\right), 2.56(\mathrm{t}, \mathrm{J}=6.2 \mathrm{~Hz}$, $\left.2 \mathrm{H}, \mathrm{CH}_{2}-3^{\prime \prime}\right), 2.32$ (s, 3H, $\left.\mathrm{CH}_{3}-7^{\prime \prime \prime}\right), 2.14$ (s, 3H, $\left.\mathrm{CH}_{3}-8^{\prime \prime \prime}\right) ; \quad \operatorname{EIMS}(\mathrm{m} / \mathrm{z}): 355 \quad[\mathrm{M}]^{+}, 244$ $\left[\mathrm{C}_{15} \mathrm{H}_{20} \mathrm{~N}_{2} \mathrm{O}\right]^{*+}, \quad 231 \quad\left[\mathrm{C}_{14} \mathrm{H}_{19} \mathrm{~N}_{2} \mathrm{O}\right]^{+}, \quad 193$ $\left[\mathrm{C}_{10} \mathrm{H}_{13} \mathrm{~N}_{2} \mathrm{O}_{2}\right]^{+}, 175\left[\mathrm{C}_{11} \mathrm{H}_{13} \mathrm{NO}^{+}, 147\left[\mathrm{C}_{9} \mathrm{H}_{9} \mathrm{NO}\right]^{++}\right.$, $138\left[\mathrm{C}_{7} \mathrm{H}_{8} \mathrm{NO}_{2}\right]^{+}, 121\left[\mathrm{C}_{8} \mathrm{H}_{11} \mathrm{~N}\right]^{++}, 95\left[\mathrm{C}_{5} \mathrm{H}_{3} \mathrm{O}_{2}\right]^{+}$.

\section{3-[4-(2-Furoyl)-1-piperazinyl]-N-(2,4- dimethylphenyl) propanamide (50)}

Pink-brown amorphous solid; yield: 92 \%; m.p: 128-130 ${ }^{\circ} \mathrm{C}$; Mol. F.: $\mathrm{C}_{20} \mathrm{H}_{25} \mathrm{~N}_{3} \mathrm{O}_{3}$; Mol. Mass: 355; IR (KBr, cm $\left.{ }^{-1}\right)$ v: $3405(\mathrm{~N}-\mathrm{H}), 3075$ (Ar C$\mathrm{H}), 2882$ (R C-H), $1646(\mathrm{C}=\mathrm{O}), 1578$ ( $\mathrm{Ar} \mathrm{C}=\mathrm{C})$, 1201 (C-O-C), 1109 (C-N-C); ${ }^{1} \mathrm{H}-\mathrm{NMR}$ (500 MHz, $\mathrm{CDCl}_{3}, \delta$ in ppm): 7.68 (br.d, $J=7.6 \mathrm{~Hz}, 1 \mathrm{H}, \mathrm{H}-$ 6"'), 7.49 (distorted d, $J=1.0 \mathrm{~Hz}, 1 \mathrm{H}, \mathrm{H}-5$ '), 7.05 (d, $J=3.5 \mathrm{~Hz}, 1 \mathrm{H}, \mathrm{H}-3$ '), 6.97 (br.d, $J=7.6 \mathrm{~Hz}$, $1 \mathrm{H}, \mathrm{H}-5^{\prime \prime \prime)}, 6.96$ (br.s, 1H, H-3"'), 6.05 (dd, $J=$ 1.7, $3.4 \mathrm{~Hz}, 1 \mathrm{H}, \mathrm{H}-4^{\prime}$ ), 3.91 (br.s, $4 \mathrm{H}, \mathrm{CH}_{2}-3$, $\left.\mathrm{CH}_{2}-5\right), 2.78$ (t, $\left.J=6.2 \mathrm{~Hz}, 2 \mathrm{H}, \mathrm{CH}_{2}-2^{\prime \prime}\right), 2.67$ (m, $\left.4 \mathrm{H}, \mathrm{CH}_{2}-2, \mathrm{CH}_{2}-4\right), 2.56$ (t, J = 6.2 Hz, 2H, CH $2^{-}$ 3"), 2.25 (s, 3H, $\left.\mathrm{CH}_{3}-7^{\prime \prime \prime}\right), 2.20$ (s, 3H, $\mathrm{CH}_{3}-8$ "'); EIMS $(\mathrm{m} / \mathrm{z}): 355[\mathrm{M}]^{+}, 244\left[\mathrm{C}_{15} \mathrm{H}_{20} \mathrm{~N}_{2} \mathrm{O}\right]^{++}, 231$ $\left[\mathrm{C}_{14} \mathrm{H}_{19} \mathrm{~N}_{2} \mathrm{O}\right]^{+}, \quad 193 \quad\left[\mathrm{C}_{10} \mathrm{H}_{13} \mathrm{~N}_{2} \mathrm{O}_{2}\right]^{+}, \quad 175$ $\left[\mathrm{C}_{11} \mathrm{H}_{13} \mathrm{NO}\right]^{+}, 147\left[\mathrm{C}_{9} \mathrm{H}_{9} \mathrm{NO}\right]^{++}, 138\left[\mathrm{C}_{7} \mathrm{H}_{8} \mathrm{NO}_{2}\right]^{+}$, $121\left[\mathrm{C}_{8} \mathrm{H}_{11} \mathrm{~N}\right]^{++}, 95\left[\mathrm{C}_{5} \mathrm{H}_{3} \mathrm{O}_{2}\right]^{+}$.

\section{3-[4-(2-Furoyl)-1-piperazinyl]-N-(2,5- dimethylphenyl) propanamide (5p)}

Off-white amorphous solid; yield: $94 \%$; m.p: 125$127{ }^{\circ} \mathrm{C}$; Mol. $\mathrm{F} .: \mathrm{C}_{20} \mathrm{H}_{25} \mathrm{~N}_{3} \mathrm{O}_{3}$; Mol. Mass: 355; IR $\left(\mathrm{KBr}, \mathrm{cm}^{-1}\right)$ v: $3412(\mathrm{~N}-\mathrm{H}), 3073(\mathrm{Ar} \mathrm{C}-\mathrm{H}), 2881$ (R C-H), 1650 (C=O), 1584 ( $\mathrm{Ar} \mathrm{C}=\mathrm{C}), 1200$ (C-OC), 1109 (C-N-C); ${ }^{1} \mathrm{H}-\mathrm{NMR}\left(500 \mathrm{MHz}^{-} \mathrm{CDCl}_{3}, \delta\right.$ in ppm): 7.78 (br.s, $\left.1 \mathrm{H}, \mathrm{H}-6^{\prime \prime \prime}\right), 7.48$ (distorted d, $\left.J=1.5 \mathrm{~Hz}, 1 \mathrm{H}, \mathrm{H}-5^{\prime}\right), 7.05(\mathrm{~d}, J=7.7 \mathrm{~Hz}, 1 \mathrm{H}, \mathrm{H}-$ 3"'), 7.04 (d, J = 3.5 Hz, 1H, H-3'), 6.87 (br.d, $J=$ $7.5 \mathrm{~Hz}, 1 \mathrm{H}, \mathrm{H}-4$ "'), 6.49 (dd, $J=1.7,3.4 \mathrm{~Hz}, 1 \mathrm{H}$, H-4'), 3.94 (br.s, 4H, CH $\left.6.2 \mathrm{~Hz}, 2 \mathrm{H}, \mathrm{CH}_{2}-2 "\right), 2.65$ (t, J = 6.2 Hz, 2H, $\mathrm{CH}_{2}-$ 3"), $2.63\left(\mathrm{~m}, 4 \mathrm{H}, \mathrm{CH}_{2}-2, \mathrm{CH}_{2}-4\right), 2.32$ (s, 3H, $\mathrm{CH}_{3}-7^{\prime \prime \prime)}$, 2.26 (s, $3 \mathrm{H}, \mathrm{CH}_{3}-8$ "'); EIMS (m/z): 355 $[\mathrm{M}]^{+}, 244\left[\mathrm{C}_{15} \mathrm{H}_{20} \mathrm{~N}_{2} \mathrm{O}\right]^{*+}, 231\left[\mathrm{C}_{14} \mathrm{H}_{19} \mathrm{~N}_{2} \mathrm{O}\right]^{+}, 193$ 
$\left[\mathrm{C}_{10} \mathrm{H}_{13} \mathrm{~N}_{2} \mathrm{O}_{2}\right]^{+}, 175\left[\mathrm{C}_{11} \mathrm{H}_{13} \mathrm{NO}\right]^{+}, 147\left[\mathrm{C}_{9} \mathrm{H}_{9} \mathrm{NO}\right]^{++}$, $138\left[\mathrm{C}_{7} \mathrm{H}_{8} \mathrm{NO}_{2}\right]^{+}, 121\left[\mathrm{C}_{8} \mathrm{H}_{11} \mathrm{~N}\right]^{++}, 95\left[\mathrm{C}_{5} \mathrm{H}_{3} \mathrm{O}_{2}\right]^{+}$.

\section{3-[4-(2-Furoyl)-1-piperazinyl]-N-(2,6- dimethylphenyl)propanamide (5q)}

Off-white amorphous solid; yield: $90 \%$; m.p.: 126-128 ${ }^{\circ} \mathrm{C}$; Mol. F.: $\mathrm{C}_{20} \mathrm{H}_{25} \mathrm{~N}_{3} \mathrm{O}_{3}$; Mol. Mass.: 355; IR $\left(\mathrm{KBr}, \mathrm{cm}^{-1}\right)$ v: $3412(\mathrm{~N}-\mathrm{H}), 3079$ (Ar C-H), 2885 (R C-H), $1655(\mathrm{C}=\mathrm{O}), 1579$ ( $\mathrm{Ar} \mathrm{C}=\mathrm{C}), 1201$ (C-O-C), 1114 (C-N-C); ${ }^{1} \mathrm{H}-\mathrm{NMR} \quad(500 \mathrm{MHz}$, $\mathrm{CDCl}_{3}, \delta$ in ppm): 7.49 (distorted $\mathrm{d}, J=1.0 \mathrm{~Hz}$, $\left.1 \mathrm{H}, \mathrm{H}-5^{\prime}\right)$, 7.06-7.01 (m, 3H, H-3'" to H-5"'), 7.05 (d, $\left.J=3.5 \mathrm{~Hz}, 1 \mathrm{H}, \mathrm{H}-3^{\prime}\right), 6.05$ (dd, $J=1.7,3.4$ $\mathrm{Hz}, 1 \mathrm{H}, \mathrm{H}-4$ '), 3.91 (br.s, 4H, $\left.\mathrm{CH}_{2}-3, \mathrm{CH}_{2}-5\right), 2.78$ (t, $\left.J=6.2 \mathrm{~Hz}, 2 \mathrm{H}, \mathrm{CH}_{2}-2 "\right), 2.67\left(\mathrm{~m}, 4 \mathrm{H}, \mathrm{CH}_{2}-2\right.$, $\mathrm{CH}_{2}-4$ ), 2.56 (t, $\left.\mathrm{J}=6.2 \mathrm{~Hz}, 2 \mathrm{H}, \mathrm{CH}_{2}-3 "\right), 2.16$ (s, $6 \mathrm{H}, \mathrm{CH}_{3}-7^{\prime \prime \prime}, \mathrm{CH}_{3}-8$ "'); EIMS (m/z): 355 [M] ${ }^{+}, 244$ $\left[\mathrm{C}_{15} \mathrm{H}_{20} \mathrm{~N}_{2} \mathrm{O}\right]^{++}, \quad 231 \quad\left[\mathrm{C}_{14} \mathrm{H}_{19} \mathrm{~N}_{2} \mathrm{O}\right]^{+}, \quad 193$ $\left[\mathrm{C}_{10} \mathrm{H}_{13} \mathrm{~N}_{2} \mathrm{O}_{2}\right]^{+}, 175\left[\mathrm{C}_{11} \mathrm{H}_{13} \mathrm{NO}^{+}, 147\left[\mathrm{C}_{9} \mathrm{H}_{9} \mathrm{NO}^{+}\right.\right.$, $138\left[\mathrm{C}_{7} \mathrm{H}_{8} \mathrm{NO}_{2}\right]^{+}, 121\left[\mathrm{C}_{8} \mathrm{H}_{11} \mathrm{~N}\right]^{++}, 95\left[\mathrm{C}_{5} \mathrm{H}_{3} \mathrm{O}_{2}\right]^{+}$.

\section{3-[4-(2-Furoyl)-1-piperazinyl]-N-(3,4- dimethylphenyl) propanamide (5r)}

White crystalline solid; yield: $89 \%$; m.p: 130-132 ${ }^{\circ} \mathrm{C}$; Mol. F.: $\mathrm{C}_{20} \mathrm{H}_{25} \mathrm{~N}_{3} \mathrm{O}_{3}$; Mol. Mass: 355; IR $\left(\mathrm{KBr}, \mathrm{cm}^{-1}\right)$ v: $3407(\mathrm{~N}-\mathrm{H}), 3076(\mathrm{Ar} \mathrm{C}-\mathrm{H}), 2879$ (R C-H), $1649(\mathrm{C}=\mathrm{O}), 1580$ ( $\mathrm{Ar} \mathrm{C}=\mathrm{C}), 1207$ (C-OC), 1105 (C-N-C); ${ }^{1} \mathrm{H}-\mathrm{NMR}\left(500 \mathrm{MHz}, \mathrm{CDCl}_{3}, \delta\right.$ in ppm): 7.49 (distorted d, $J=1.0 \mathrm{~Hz}, 1 \mathrm{H}, \mathrm{H}-5^{\prime}$ ), 7.29 (br.d, $\left.J=8.0 \mathrm{~Hz}, 1 \mathrm{H}, \mathrm{H}-6{ }^{\prime \prime \prime}\right), 7.26$ (br.s, $1 \mathrm{H}$, H-2'"), 7.05 (d, $\left.J=3.5 \mathrm{~Hz}, 1 \mathrm{H}, \mathrm{H}-3^{\prime}\right), 7.03$ (br.d, $J$ $\left.=8.0 \mathrm{~Hz}, 1 \mathrm{H}, \mathrm{H}-5{ }^{\prime \prime}\right), 6.05$ (dd, $J=1.7,3.4 \mathrm{~Hz}$, $1 \mathrm{H}, \mathrm{H}-4$ ), 3.91 (br.s, $4 \mathrm{H}, \mathrm{CH}_{2}-3, \mathrm{CH}_{2}-5$ ), 2.78 (t, $\left.J=6.2 \mathrm{~Hz}, 2 \mathrm{H}, \mathrm{CH}_{2}-2 "\right), 2.67\left(\mathrm{~m}, 4 \mathrm{H}, \mathrm{CH}_{2}-2\right.$, $\mathrm{CH}_{2}-4$ ), 2.56 (t, $\left.J=6.2 \mathrm{~Hz}, 2 \mathrm{H}, \mathrm{CH}_{2}-3 "\right), 2.21$ (s, $\left.3 \mathrm{H}, \mathrm{CH}_{3}-7^{\prime \prime \prime}\right), 2.18\left(\mathrm{~s}, 3 \mathrm{H}, \mathrm{CH}_{3}-8^{\prime \prime \prime}\right)$; $\operatorname{EIMS}(\mathrm{m} / \mathrm{z})$ : $355[\mathrm{M}]^{+}, 244\left[\mathrm{C}_{15} \mathrm{H}_{20} \mathrm{~N}_{2} \mathrm{O}\right]^{+}, 231\left[\mathrm{C}_{14} \mathrm{H}_{19} \mathrm{~N}_{2} \mathrm{O}\right]^{+}$, $193 \quad\left[\mathrm{C}_{10} \mathrm{H}_{13} \mathrm{~N}_{2} \mathrm{O}_{2}\right]^{+}, \quad 175 \quad\left[\mathrm{C}_{11} \mathrm{H}_{13} \mathrm{NO}\right]^{+}, \quad 147$ $\left[\mathrm{C}_{9} \mathrm{H}_{9} \mathrm{NO}\right]^{+}, 138\left[\mathrm{C}_{7} \mathrm{H}_{8} \mathrm{NO}_{2}\right]^{+}, 121\left[\mathrm{C}_{8} \mathrm{H}_{11} \mathrm{~N}\right]^{++}, 95$ $\left[\mathrm{C}_{5} \mathrm{H}_{3} \mathrm{O}_{2}\right]^{+}$.

\section{3-[4-(2-Furoyl)-1-piperazinyl]-N-(3,5- dimethylphenyl) propanamide (5s)}

Light-brown amorphous solid; yield: $93 \%$; m.p.: 128-130 ${ }^{\circ} \mathrm{C}$; Mol. F.: $\mathrm{C}_{20} \mathrm{H}_{25} \mathrm{~N}_{3} \mathrm{O}_{3}$; Mol. Mass: 355; IR (KBr, cm $\left.{ }^{-1}\right)$ v: 3409 (N-H), 3070 (Ar C-H), $2886(\mathrm{R} \mathrm{C}-\mathrm{H}), 1646(\mathrm{C}=\mathrm{O}), 1575(\mathrm{Ar} \mathrm{C}=\mathrm{C}), 1208$ (C-O-C), 1117 (C-N-C); ${ }^{1} \mathrm{H}-\mathrm{NMR}(500 \mathrm{MHz}$, $\mathrm{CDCl}_{3}, \delta$ in ppm): 7.49 (distorted d, $J=1.0 \mathrm{~Hz}$, $\left.1 \mathrm{H}, \mathrm{H}-5^{\prime}\right), 7.15$ (br.s, 2H, H-2"', H-6"'), 7.05 (d, J $=3.5 \mathrm{~Hz}, 1 \mathrm{H}, \mathrm{H}-3^{\prime}$ ), 6.73 (br.s, $\left.1 \mathrm{H}, \mathrm{H}-4^{\prime \prime \prime}\right), 6.05$ (dd, $J=1.7,3.4 \mathrm{~Hz}, 1 \mathrm{H}, \mathrm{H}-4$ '), 3.91 (br.s, $4 \mathrm{H}$, $\left.\mathrm{CH}_{2}-3, \mathrm{CH}_{2}-5\right), 2.78$ (t, $\left.J=6.2 \mathrm{~Hz}, 2 \mathrm{H}, \mathrm{CH}_{2}-2 "\right)$, $2.67\left(\mathrm{~m}, 4 \mathrm{H}, \mathrm{CH}_{2}-2, \mathrm{CH}_{2}-4\right), 2.56(\mathrm{t}, \mathrm{J}=6.2 \mathrm{~Hz}$, $\left.2 \mathrm{H}, \mathrm{CH}_{2}-3 "\right), 2.26$ (s, 6H, $\mathrm{CH}_{3}-7^{\prime \prime \prime}, \mathrm{CH}_{3}-8$ "'); EIMS $(\mathrm{m} / \mathrm{z}): \quad 355 \quad[\mathrm{M}]^{+}, \quad 244 \quad\left[\mathrm{C}_{15} \mathrm{H}_{20} \mathrm{~N}_{2} \mathrm{O}\right]^{*+}, \quad 231$
$\left[\mathrm{C}_{14} \mathrm{H}_{19} \mathrm{~N}_{2} \mathrm{O}\right]^{+}, \quad 193 \quad\left[\mathrm{C}_{10} \mathrm{H}_{13} \mathrm{~N}_{2} \mathrm{O}_{2}\right]^{+}, \quad 175$ $\left[\mathrm{C}_{11} \mathrm{H}_{13} \mathrm{NO}\right]^{+}, 147\left[\mathrm{C}_{9} \mathrm{H}_{9} \mathrm{NO}\right]^{+}, 138\left[\mathrm{C}_{7} \mathrm{H}_{8} \mathrm{NO}_{2}\right]^{+}$, $121\left[\mathrm{C}_{8} \mathrm{H}_{11} \mathrm{~N}\right]^{*+}, 95\left[\mathrm{C}_{5} \mathrm{H}_{3} \mathrm{O}_{2}\right]^{+}$.

\section{3-[4-(2-Furoyl)-1-piperazinyl]-N-(2-ethyl-6- methylphenyl) propanamide (5t)}

Red-brown, sticky amorphous solid; yield: $90 \%$; m.p: $131-133{ }^{\circ} \mathrm{C}$; Mol. F.: $\mathrm{C}_{21} \mathrm{H}_{27} \mathrm{~N}_{3} \mathrm{O}_{3}$; Mol. Mass: 369; IR $\left(\mathrm{KBr}, \mathrm{cm}^{-1}\right)$ v: $3406(\mathrm{~N}-\mathrm{H}), 3089$ (Ar C-H), $2887(\mathrm{R} \mathrm{C}-\mathrm{H}), 1655(\mathrm{C}=\mathrm{O}), 1588(\mathrm{Ar}$ $\mathrm{C}=\mathrm{C}), 1198$ (C-O-C), 1110 (C-N-C); ${ }^{1} \mathrm{H}-\mathrm{NMR}$ (500 MHz, $\mathrm{CDCl}_{3}, \delta$ in ppm): 7.49 (distorted d, $J$ $\left.=1.0 \mathrm{~Hz}, 1 \mathrm{H}, \mathrm{H}-5^{\prime}\right), 7.05\left(\mathrm{~d}, J=3.5 \mathrm{~Hz}, 1 \mathrm{H}, \mathrm{H}-3^{\prime}\right)$, 6.98-7.14 (m, 3H, H-3"' to $\left.\mathrm{H}-5^{\prime \prime \prime}\right), 6.05$ (dd, $J=$ 1.7, 3.4 Hz, $\left.1 \mathrm{H}, \mathrm{H}-4^{\prime}\right), 3.91$ (br.s, $4 \mathrm{H}, \mathrm{CH}_{2}-3$, $\mathrm{CH}_{2}-5$ ), 2.78 (t, $\left.J=6.2 \mathrm{~Hz}, 2 \mathrm{H}, \mathrm{H}-2 "\right), 2.67(\mathrm{~m}$, $\left.4 \mathrm{H}, \mathrm{CH}_{2}-2, \mathrm{CH}_{2}-4\right), 2.56$ (t, $J=6.2 \mathrm{~Hz}, 2 \mathrm{H}, \mathrm{CH}_{2}-$ 3"), 2.46 (q, $\left.J=7.5 \mathrm{~Hz}, 2 \mathrm{H}, \mathrm{CH}_{2}-7^{\prime \prime \prime}\right), 1.97$ (s, 3H, $\left.\mathrm{CH}_{3}-9^{\prime \prime \prime}\right), 1.02$ (t, $J=7.5,3 \mathrm{H}, \mathrm{CH}_{3}-8{ }^{\prime \prime \prime)}$; EIMS $(\mathrm{m} / \mathrm{z}): 369[\mathrm{M}]^{+},\left[\mathrm{C}_{11} \mathrm{H}_{15} \mathrm{~N}_{2} \mathrm{O}_{2}\right]^{+}, 258\left[\mathrm{C}_{16} \mathrm{H}_{22} \mathrm{~N}_{2} \mathrm{O}\right]^{++}$, $245 \quad\left[\mathrm{C}_{15} \mathrm{H}_{21} \mathrm{~N}_{2} \mathrm{O}\right]^{+}, \quad 193 \quad\left[\mathrm{C}_{10} \mathrm{H}_{13} \mathrm{~N}_{2} \mathrm{O}_{2}\right]^{+}, 189$ $\left[\mathrm{C}_{12} \mathrm{H}_{15} \mathrm{NO}\right]^{+}, 161\left[\mathrm{C}_{10} \mathrm{H}_{11} \mathrm{NO}\right]^{++}, 138\left[\mathrm{C}_{7} \mathrm{H}_{8} \mathrm{NO}_{2}\right]^{+}$, $135\left[\mathrm{C}_{9} \mathrm{H}_{13} \mathrm{~N}\right]^{*+}, 95\left[\mathrm{C}_{5} \mathrm{H}_{3} \mathrm{O}_{2}\right]^{+}$.

\section{3-[4-(2-Furoyl)-1-piperazinyl]-N-(5-chloro-2- methoxyphenyl) propanamide (5u)}

Light-brown amorphous solid; yield: 90 \%; m.p.: 112-114 ${ }^{\circ} \mathrm{C}$; Mol. F..: $\mathrm{C}_{19} \mathrm{H}_{22} \mathrm{ClN}_{3} \mathrm{O}_{4}$; Mol. Mass: 391; IR ( $\left.\mathrm{KBr}, \mathrm{cm}^{-1}\right)$ v: $3407(\mathrm{~N}-\mathrm{H}), 3089(\mathrm{Ar} \mathrm{C}-\mathrm{H})$, $2884(\mathrm{R} \mathrm{C}-\mathrm{H}), 1655(\mathrm{C}=\mathrm{O}), 1584(\mathrm{Ar} \mathrm{C}=\mathrm{C}), 1197$ (C-O-C), 1109 (C-N-C); ${ }^{1} \mathrm{H}-\mathrm{NMR}(500 \mathrm{MHz}$, $\mathrm{CDCl}_{3}, \delta$ in ppm): $8.52\left(\mathrm{~d}, J=2.1 \mathrm{~Hz}, 1 \mathrm{H}, \mathrm{H}-6{ }^{\prime \prime \prime}\right)$, 7.49 (distorted d, $\left.J=1.0 \mathrm{~Hz}, 1 \mathrm{H}, \mathrm{H}-5^{\prime}\right), 7.05$ (d, $J$ $\left.=3.5 \mathrm{~Hz}, 1 \mathrm{H}, \mathrm{H}-3^{\prime}\right), 7.01$ (dd, $J=8.5,2.1 \mathrm{~Hz}, \mathrm{H}-$ 4"'), 6.79 (d, $J=8.5 \mathrm{~Hz}, \mathrm{H}-3$ '"), 6.05 (dd, $J=1.7$, $\left.3.4 \mathrm{~Hz}, 1 \mathrm{H}, \mathrm{H}-4^{\prime}\right), 3.92$ (s, 3H, $\left.\mathrm{H}_{3} \mathrm{C}-8^{\prime \prime \prime}\right), 3.91$ (br.s, $\left.4 \mathrm{H}, \mathrm{CH}_{2}-3, \mathrm{CH}_{2}-5\right), 2.78(\mathrm{t}, J=6.2 \mathrm{~Hz}, 2 \mathrm{H}$, $\mathrm{CH}_{2}-2$ "), $2.67\left(\mathrm{~m}, 4 \mathrm{H}, \mathrm{CH}_{2}-2, \mathrm{CH}_{2}-4\right), 2.56$ (t, $\mathrm{J}=$ $\left.6.2 \mathrm{~Hz}, 2 \mathrm{H}, \mathrm{CH}_{2}-3 "\right)$; $\operatorname{EIMS}(\mathrm{m} / \mathrm{z}): 393[\mathrm{M}+2]^{+}$,

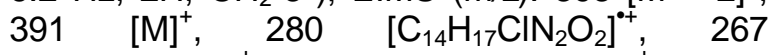
$\left[\begin{array}{lll}\left.\mathrm{C}_{13} \mathrm{H}_{16} \mathrm{CIN}_{2} \mathrm{O}_{2}\right]^{+}, & 211 \quad\left[\mathrm{C}_{10} \mathrm{H}_{10} \mathrm{ClNO}_{2}\right]^{+}, & 193\end{array}\right.$ $\left[\begin{array}{llll}\left.\mathrm{C}_{10} \mathrm{H}_{13} \mathrm{~N}_{2} \mathrm{O}_{2}\right]^{+}, & 183 & {\left[\mathrm{C}_{8} \mathrm{H}_{6} \mathrm{ClNO}_{2}\right]^{+},} & 157\end{array}\right.$ $\left[\mathrm{C}_{7} \mathrm{H}_{6} \mathrm{CINO}\right]^{+}, 138\left[\mathrm{C}_{7} \mathrm{H}_{8} \mathrm{NO}_{2}\right]^{+}, 95\left[\mathrm{C}_{5} \mathrm{H}_{3} \mathrm{O}_{2}\right]^{+}$.

\section{Antibacterial and hemolytic activities}

All synthesized molecules were screened against Gram-positive and Gram-negative bacteria and were found to be good to excellent inhibitors. The results are given as \% inhibition and MIC values in Tables 1 and 2. The \% hemolysis of the synthesized compounds is also given in Table 2. The results for compounds were compared with that for Triton X-100 and phosphate-buffered saline (PBS). 
Table 1: Antibacterial activity (\%, inhibition) of the synthesized compounds

\begin{tabular}{|c|c|c|c|c|c|}
\hline \multirow{2}{*}{ Compound } & \multicolumn{5}{|c|}{ Inhibition (\%) } \\
\hline & S. typhi (-) & E. coli (-) & P. aeruginosa (-) & B. subtilis (+) & S. aureus (+) \\
\hline $5 a$ & $81.75 \pm 0.40$ & $60.65 \pm 0.05$ & $65.70 \pm 0.20$ & $68.15 \pm 0.35$ & $75.53 \pm 0.60$ \\
\hline $5 b$ & $75.87 \pm 0.73$ & $64.80 \pm 0.70$ & $75.39 \pm 0.04$ & $66.60 \pm 0.50$ & $60.85 \pm 0.15$ \\
\hline $5 c$ & $73.00 \pm 0.60$ & $65.05 \pm 0.75$ & $77.21 \pm 0.68$ & $64.05 \pm 0.45$ & $64.00 \pm 0.48$ \\
\hline $5 d$ & $73.20 \pm 0.80$ & $61.45 \pm 0.65$ & $71.79 \pm 0.71$ & $68.65 \pm 0.65$ & $64.00 \pm 0.30$ \\
\hline $5 e$ & $81.37 \pm 0.77$ & $69.70 \pm 0.50$ & $68.46 \pm 0.35$ & $66.85 \pm 0.15$ & $86.30 \pm 0.50$ \\
\hline $5 f$ & $83.20 \pm 0.60$ & $61.15 \pm 0.25$ & $59.36 \pm 0.89$ & $72.85 \pm 0.85$ & $68.80 \pm 0.65$ \\
\hline $5 g$ & $84.83 \pm 0.82$ & $80.15 \pm 0.05$ & $69.86 \pm 0.07$ & $61.15 \pm 0.85$ & $86.35 \pm 0.65$ \\
\hline $5 h$ & $78.23 \pm 0.37$ & $72.75 \pm 1.00$ & $67.93 \pm 0.36$ & $71.20 \pm 0.10$ & $84.55 \pm 0.15$ \\
\hline $5 i$ & $82.67 \pm 0.07$ & $65.25 \pm 0.95$ & $43.57 \pm 0.50$ & $70.55 \pm 0.25$ & $79.80 \pm 0.30$ \\
\hline $5 j$ & $85.73 \pm 0.40$ & $64.40 \pm 0.45$ & $47.00 \pm 0.34$ & $70.65 \pm 0.85$ & $77.50 \pm 0.90$ \\
\hline $5 k$ & $80.00 \pm 0.33$ & $62.90 \pm 0.60$ & $61.21 \pm 0.36$ & $63.30 \pm 0.60$ & $70.30 \pm 0.80$ \\
\hline $5 I$ & $79.07 \pm 0.17$ & $74.71 \pm 0.01$ & $70.90 \pm 0.12$ & $60.15 \pm 0.25$ & $78.90 \pm 0.15$ \\
\hline $5 m$ & $79.63 \pm 0.83$ & $56.80 \pm 0.56$ & $57.39 \pm 0.82$ & $67.80 \pm 0.58$ & $66.75 \pm 0.45$ \\
\hline $5 n$ & $75.93 \pm 1.00$ & $62.60 \pm 0.50$ & $68.46 \pm 0.75$ & $69.65 \pm 0.95$ & $66.00 \pm 0.43$ \\
\hline 50 & $71.70 \pm 0.37$ & $58.65 \pm 0.05$ & $63.18 \pm 0.96$ & $64.65 \pm 0.35$ & $56.25 \pm 0.55$ \\
\hline $5 p$ & $73.70 \pm 0.57$ & $60.20 \pm 0.20$ & $82.71 \pm 0.21$ & $67.25 \pm 0.85$ & $62.15 \pm 0.05$ \\
\hline $5 q$ & $75.87 \pm 0.60$ & $64.70 \pm 0.14$ & $51.32 \pm 0.18$ & $66.90 \pm 0.60$ & $62.60 \pm 0.25$ \\
\hline $5 r$ & $82.80 \pm 078$ & $67.95 \pm 0.85$ & $70.29 \pm 0.50$ & $60.20 \pm 0.80$ & $78.85 \pm 0.05$ \\
\hline $5 s$ & $82.00 \pm 0.34$ & $71.05 \pm 0.55$ & $72.93 \pm 0.21$ & $65.50 \pm 0.60$ & $72.05 \pm 0.75$ \\
\hline $5 t$ & $78.83 \pm 0.57$ & $52.85 \pm 0.85$ & $35.00 \pm 0.71$ & $57.00 \pm 0.50$ & $83.85 \pm 0.35$ \\
\hline $5 u$ & $81.40 \pm 0.60$ & $60.00 \pm 0.30$ & $38.36 \pm 0.07$ & $64.60 \pm 0.30$ & $73.30 \pm 0.34$ \\
\hline Ciprofloxacin & $91.05 \pm 0.68$ & $92.32 \pm 0.42$ & $92.50 \pm 0.34$ & $92.02 \pm 053$ & $91.44 \pm 0.64$ \\
\hline
\end{tabular}

Table 2: Antibacterial activity (MIC) and hemolytic activity of the synthesized compounds

\begin{tabular}{|c|c|c|c|c|c|c|}
\hline \multirow[b]{2}{*}{ Compound } & \multicolumn{5}{|c|}{ MIC $(\mu \mathrm{M})$} & \multirow{2}{*}{$\begin{array}{c}\text { Hemolytic } \\
\text { activity } \\
\%\end{array}$} \\
\hline & S. typhi (-) & E. coli (-) & $\begin{array}{c}P . \\
\underset{(-)}{\text { aeruginosa }}\end{array}$ & $\begin{array}{c}\text { B. subtilis } \\
(+)\end{array}$ & $\begin{array}{c}\text { S. aureus } \\
(+)\end{array}$ & \\
\hline $5 a$ & $8.37 \pm 0.12$ & $12.92 \pm 0.11$ & $10.29 \pm 0.30$ & $9.97 \pm 0.77$ & $9.22 \pm 0.76$ & 15.48 \\
\hline $5 b$ & $9.65 \pm 0.17$ & $12.87 \pm 0.51$ & $9.19 \pm 0.52$ & $10.21 \pm 0.44$ & $14.28 \pm 0.75$ & 8.41 \\
\hline $5 c$ & $9.87 \pm 0.45$ & $12.76 \pm 0.90$ & $8.97 \pm 0.10$ & $10.13 \pm 0.89$ & $12.46 \pm 0.50$ & 61.93 \\
\hline $5 d$ & $9.74 \pm 0.13$ & $15.31 \pm 0.78$ & $9.46 \pm 0.13$ & $10.22 \pm 0.48$ & $11.97 \pm 0.73$ & 3.98 \\
\hline $5 e$ & $9.28 \pm 0.63$ & $10.49 \pm 0.64$ & $9.96 \pm 0.49$ & $10.32 \pm 0.24$ & $8.65 \pm 0.50$ & 3.12 \\
\hline $5 f$ & $9.41 \pm 0.23$ & $14.72 \pm 0.10$ & $15.98 \pm 0.10$ & $9.76 \pm 0.15$ & $10.53 \pm 0.30$ & 35.56 \\
\hline $5 g$ & $8.45 \pm 0.31$ & $8.97 \pm 0.12$ & $9.89 \pm 0.30$ & $13.52 \pm 0.12$ & $8.34 \pm 0.55$ & 8.03 \\
\hline $5 h$ & $9.12 \pm 0.23$ & $9.52 \pm 0.75$ & $10.43 \pm 0.74$ & $9.47 \pm 0.73$ & $8.73 \pm 0.90$ & 64.18 \\
\hline $5 i$ & $8.41 \pm 0.11$ & $10.87 \pm 0.19$ & - & $9.24 \pm 0.50$ & $8.93 \pm 0.64$ & 5.52 \\
\hline $5 j$ & $8.68 \pm 0.17$ & $14.71 \pm 0.31$ & $9.85 \pm 0.28$ & $10.61 \pm 0.10$ & $10.15 \pm 0.30$ & 4.78 \\
\hline $5 k$ & $8.97 \pm 0.27$ & $14.73 \pm 0.75$ & $13.57 \pm 0.59$ & $12.63 \pm 0.97$ & $9.76 \pm 0.12$ & 8.41 \\
\hline $5 I$ & $9.10 \pm 0.43$ & $16.58 \pm 0.80$ & $8.67 \pm 0.94$ & $9.29 \pm 0.89$ & $13.12 \pm 0.37$ & 3.93 \\
\hline $5 m$ & $9.11 \pm 0.24$ & $15.75 \pm 0.39$ & $15.97 \pm 0.44$ & $12.75 \pm 0.27$ & $10.46 \pm 0.51$ & 7.61 \\
\hline $5 n$ & $9.56 \pm 0.38$ & $14.75 \pm 0.19$ & $9.85 \pm 0.56$ & $9.87 \pm 0.50$ & $10.53 \pm 0.53$ & 15.88 \\
\hline 50 & $10.23 \pm 0.87$ & $16.79 \pm 0.45$ & $12.42 \pm 0.40$ & $12.87 \pm 0.14$ & $16.95 \pm 0.45$ & 2.97 \\
\hline $5 p$ & $10.01 \pm 0.49$ & $15.89 \pm 0.79$ & $8.65 \pm 0.57$ & $10.24 \pm 0.87$ & $14.75 \pm 0.70$ & 4.35 \\
\hline $5 q$ & $9.44 \pm 0.39$ & $12.65 \pm 0.33$ & $19.85 \pm 0.10$ & $10.67 \pm 0.50$ & $13.74 \pm 0.60$ & 4.30 \\
\hline $5 r$ & $8.98 \pm 0.54$ & $9.80 \pm 0.41$ & $9.34 \pm 0.56$ & $11.54 \pm 0.23$ & $9.65 \pm 0.34$ & 10.87 \\
\hline $5 s$ & $8.68 \pm 0.64$ & $10.89 \pm 0.23$ & $9.78 \pm 0.42$ & $14.32 \pm 0.61$ & $8.98 \pm 0.23$ & 15.89 \\
\hline $5 t$ & $9.34 \pm 0.50$ & $17.42 \pm 0.15$ & - & $15.98 \pm 0.28$ & $8.85 \pm 0.12$ & 20.08 \\
\hline $5 u$ & $8.70 \pm 0.98$ & $16.32 \pm 0.71$ & - & $11.85 \pm 0.52$ & $9.53 \pm 0.89$ & 12.89 \\
\hline $\begin{array}{l}\text { Ciprofloxacin } \\
\text { PBS }\end{array}$ & $7.45 \pm 0.58$ & $7.16 \pm 0.58$ & $7.14 \pm 0.18$ & $7.29 \pm 0.90$ & $7.80 \pm 0.19$ & 0.09 \\
\hline Triton $X-100$ & & & & & & 100 \\
\hline
\end{tabular}

Note: Minimum inhibitory concentration (MIC) was calculated using different concentrations (ranging 5 - 30 $\mu \mathrm{g} /$ well) and EZ-Fit software (Perrella Scientific Inc. Amherst, NH, USA) 


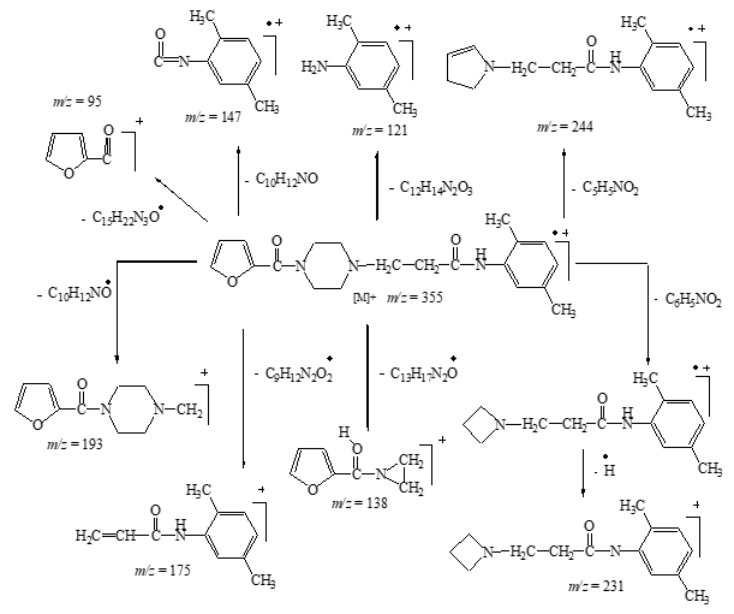

Figure 2: Mass fragmentation pattern of $5 p$

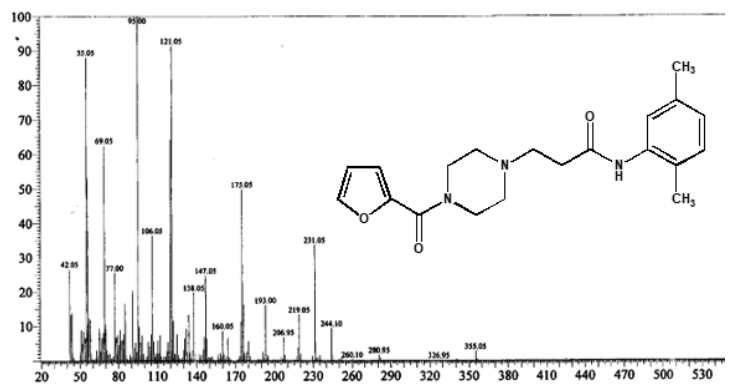

Figure 3: EIMS spectrum of 5p

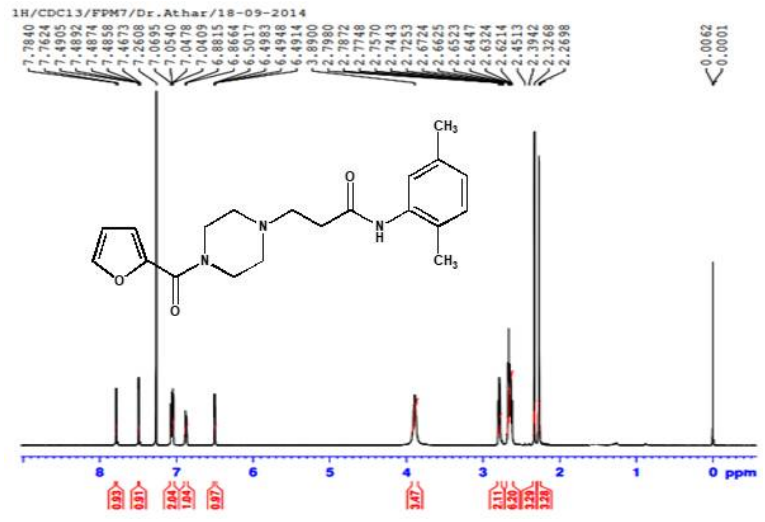

Figure 4: ${ }^{1} \mathrm{H}-\mathrm{NMR}$ spectrum of $5 \mathrm{p}$

\section{DISCUSSION}

Some

(substituted)

synthesized antibacterial agents with mild cytotoxicity. The synthesis has been outlined in scheme 1 and structures of these targeted molecules are shown in Figure 1. The procedures of synthesis and conditions of reactions are described in the experimental section. The structural assignment of one of the compounds is described hereby for clear understanding of interpretational decorum of the synthesized compounds.
Compound $5 \mathrm{p}$ was obtained as an amorphous solid having an off-white color and melting point of $125-127{ }^{\circ} \mathrm{C}$ with Mol. F. of $\mathrm{C}_{20} \mathrm{H}_{25} \mathrm{~N}_{3} \mathrm{O}_{3}$, corroborated through $\mathrm{El}-\mathrm{MS}$ with $[\mathrm{M}]^{+}$peak of 355. The fragment of $N$-furoyl group appeared at $\mathrm{m} / \mathrm{z} 95$ and that of 2,5-dimethylaniline moiety at $m / z 121$.

The IR spectrum displayed a list of peaks for dominant functionalities of the amidic carbonyl group and furoylpiperazine at $3412(\mathrm{~N}-\mathrm{H}), 3073$ (Ar C-H), 2881 (R C-H), 1650 (C=O), 1584 (Ar $\mathrm{C}=\mathrm{C}), 1200$ (C-O-C) and 1109 (C-N-C). In the aromatic region of ${ }^{1} \mathrm{H}-\mathrm{NMR}$ spectrum, three signals resonating at $\delta 7.78$ (br.s, $\left.1 \mathrm{H}, \mathrm{H}-6^{\prime \prime \prime}\right)$, $7.05\left(\mathrm{~d}, J=7.7 \mathrm{~Hz}, 1 \mathrm{H}, \mathrm{H}-3^{\prime \prime \prime}\right)$ and 6.87 (br.d, $J=$ $7.5 \mathrm{~Hz}, 1 \mathrm{H}, \mathrm{H}-4$ "') $^{2}$ were typical for the 2,5dimethylphenyl ring. Three protons of the furan ring resonated at $\delta 7.48$ (distorted $\mathrm{d}, J=1.5 \mathrm{~Hz}$, $\left.1 \mathrm{H}, \mathrm{H}-5^{\prime}\right), 7.04\left(\mathrm{~d}, J=3.5 \mathrm{~Hz}, 1 \mathrm{H}, \mathrm{H}-3^{\prime}\right)$ and 6.49 (dd, $J=1.7,3.4 \mathrm{~Hz}, 1 \mathrm{H}, \mathrm{H}-4^{\prime}$ ). In the aliphatic region of the ${ }^{1} \mathrm{H}-\mathrm{NMR}$ spectrum, two signals resonated at $\delta 3.94$ (br.s, $4 \mathrm{H}, \mathrm{CH}_{2}-3, \mathrm{CH}_{2}-5$ ) and 2.64-2.62 (m, 4H, $\left.\mathrm{CH}_{2}-2, \mathrm{CH}_{2}-4\right)$ for the piperazine ring; two signals at $\delta 2.77(\mathrm{t}, J=6.2$ $\mathrm{Hz}, 2 \mathrm{H}, \mathrm{CH}_{2}-2^{\prime \prime}$ ) and 2.65 (t, $\mathrm{J}=6.2 \mathrm{~Hz}, 2 \mathrm{H}, \mathrm{CH}_{2}-$ 3") for two methylene groups of propanamide; and two signals at $\delta 2.32\left(\mathrm{~s}, 3 \mathrm{H}, \mathrm{CH}_{3}-7{ }^{\prime \prime \prime}\right)$ and 2.26 (s, 3H, $\mathrm{CH}_{3}-8$ "') for two methyl groups attached to the aromatic ring at positions 2 and 5. The ${ }^{1} \mathrm{H}$-NMR spectrum of $5 p$ is shown in Figure $\underline{4}$. The synthesized molecule was designated 3-(4-(furan-2-carbonyl) piperazin-1yl)-N-(2,5-dimethylphenyl) propanamide, and other compounds were also structurally elucidated.

The different synthesized 3-[4-(2-furoyl)-1piperazinyl]- $N$-(substituted) propanamides showed notable activity against both Grampositive and Gram-negative bacterial strains compared to ciprofloxacin. A comparison of the two compounds $\mathbf{5 a}$ bearing an unsubstituted phenyl group and $5 \mathbf{l}$ bearing an aliphatic cyclohexyl group showed that 5 a was more active against $S$. typhi, $E$. coli and $S$. aureus, but 5I, more against $P$. aeruginosa and $B$. subtilis. The presence of an aromatic system at the nitrogen of the propanamoyl group had a positive effect on the bioactivity of the synthesized molecule. The hemolysis results for compound $5 a$ showed that it is more toxic than 51 . The increase in the distance of the unsubstituted phenyl ring from the nitrogen of the amidic group resulted in an increase in bioactivity potential but this effect was found to be the reverse for a too long aliphatic chain. Therefore, compound $5 \mathrm{e}$ bearing a benzyl group was found to be more 
active than 5 a bearing a phenyl group, but $5 f$ bearing a phenylethyl group was the least active.

Furthermore, 5e was the least toxic compound among these three. No considerable difference in bioactivity potential was found between the three compounds $\mathbf{5 b} \mathbf{b} \mathbf{d}$, bearing a methyl-substituted phenyl group at the ortho, meta and para positions. When a 2-methylphenyl group (compound 5b) was replaced by a 2-ethylphenyl group (compound $\mathbf{5 g}$ ), the bioactivity potential was found to be increased and also with low toxicity. The presence of an ethoxy group at the ortho position of the phenyl ring (5i) did not favor higher bioactivity.

A similar order of bioactivity was found for 4methylphenyl (compound 5d) and 4-ethylphenyl (compound $5 \mathrm{~h}$ ), but $5 \mathrm{~h}$ was more toxic. 4Ethoxyphenyl (compound $\mathbf{5 j}$ ) had a little less antibacterial activity compared to $5 \mathrm{~h}$ but it possessed much less toxicity. This shows that the substitution of ethyl at the ortho position of the phenyl ring (compound $\mathbf{5 g}$ ) resulted in more activity with low toxicity as compared to that at the para position (compound 5h). A similar pattern of bioactivity was observed for the ethoxy group at the ortho and para positions. Among those with dimethylphenyl groups, compounds $5 n-q$, bearing one methyl at the ortho position and a second at other positions turned out to be less effective compared to compounds $5 r$ and $\mathbf{5 s}$, bearing one methyl at the meta position and a second at other positions. Among compounds $\mathbf{5 n - q}, \mathbf{5 n}$ was the most active, but it showed high toxicity. The other three showed comparable potential, with no considerable effect of variation in position of substitution. Both compounds $5 r$ and $\mathbf{5 s}$ also showed a similar order of activity.

Overall, all synthesized molecules showed impressive antibacterial activity against $S$. typhi. The aromatic rings were responsible for more interaction, and the alkyl groups in better positions accounted for higher antibacterial activity. The compounds exhibiting low toxicity could be further subjected to in vivo study to assess their application in a drug design program.

\section{CONCLUSION}

The findings of this study show that almost all the derivatives are active against all the strains of Gram-positive and Gram-negative bacteria tested. Only a few of the synthesized molecules possess moderate cytotoxicity. Thus, these molecules have some potential as therapeutic agents, but further studies are required in this regard.

\section{DECLARATIONS}

\section{Acknowledgement}

The authors acknowledge the Higher Education Commission of Pakistan for financial support. Dr. A. Leyva (USA) provided English editing of the manuscript.

\section{Conflict of interest}

The authors declare that they have no conflict of interest with regard to this study.

\section{Contribution of authors}

We declare that this work was performed by the authors named in this article and all liabilities pertaining to claims relating to the content of this article will be borne by the authors. Ghulam Hussain synthesized and characterized all the compounds presented in this research paper. Muhammad Athar Abbasi supervised the project. Aziz-ur-Rehman and Sabahat Zahra Siddiqui are co-workers in this project. Rabia Malik evaluated the antibacterial activity of the synthesized molecules, under the supervision of Irshad Ahmad. Zahid Mushtaq determined the hemolytic activity of the synthesized molecules, under the supervision of Muhammad Shahid. Syed Adnan Ali Shah evaluated the spectral data of the synthesized compounds.

\section{REFERENCES}

1. Han $M$, Han $Y$, Song $C$, Hahn, $H$. The design and synthesis of 1,4-substituted piperazine derivatives as triple reuptake inhibitors. Bull Korean Chem Soc 2012; 33(8): 2597-2602.

2. Dou D, He G, Mandadapu SR, Aravapalli S, Kim Y, Chang K, Groutas WC. Inhibition of noroviruses by piperazine derivatives. Bioorg Med Chem Lett 2012; 22: 377-379.

3. Moir EM, Yoshiizumi K, Cairns J, Cowley P, Ferguson M, Jeremiah F, Kiyoi $T$, Morphy R, Tierney J, Wishart G, York M, Baker J, Cottney JE, Houghton AK, McPhail P, Osprey A, Walker G, Adam JM. Design, synthesis, and structure-activity relationship study of bicyclic piperazine analogs of indole-3-carboxamides as novel cannabinoid CB1 receptor agonists. Bioorg Med Chem Lett 2010; 20: 7327-7330.

4. Nozawa D, Okubo T, Ishii T, Kakinuma H, Chaki S, Okuyama S, Nakazato A. Structure-activity relationships of novel piperazines as antagonists for the melanocortin-4 receptor. Bioorg Med Chem 2007; 15: 1989-2005. 
5. Chaudhary $P$, Kumar $R$, Verma $A K$, Singh $D$, Yadav $V$, Chhillar AK, Sharmab GL, Chandra R. Synthesis and antimicrobial activity of $\mathrm{N}$-alkyl and $\mathrm{N}$-aryl piperazine derivatives. Bioorg Med Chem 2006; 14: 1819-1826.

6. Sadashiva CT, Chandra JNNS, Ponnappa KC, Gowdab TV, Rangappa KS. Synthesis and efficacy of 1-[bis(4fluorophenyl)-methyl]piperazine derivatives for acetylcholinesterase inhibition, as a stimulant of central cholinergic neurotransmission in Alzheimer's disease. Bioorg Med Chem Lett 2006; 16: 3932-3936.

7. Bru'der P, Grimstvedt A, Mejdell T, Svendsen HF. CO2 capture into aqueous solutions of piperazine activated 2amino-2-methyl-1-propanol. Chem Eng Sci 2011; 66: 6193-6198.

8. Yamaguchi I, Kado A, Fukuda $T$, Fukumoto $H$, Yamamoto $T$, Sato $M$. Ionic polymers and oligomers with expanded $\pi$-conjugation system derived from through-space interaction in piperazinium ring. Eur Polym J 2010; 46: 1119-1130.

9. Autore G, Caruso A, Marzocco S, Nicolaus B, Palladino C, Pinto A, Popolo A, Sinicropi MS, Tommonaro G, Saturnino C. Acetamide derivatives with antioxidant activity and potential, anti-inflammatory activity. Molecules 2010; 15: 2028-2038.
10. Ayhan-Kılcıgil G, Gurkan S, Coban T, Ozdamar ED, CanEke B. Synthesis and evaluation of antioxidant properties of novel 2-[2-(4-chlorophenyl) benzimidazole1-yl]-N-(2-arylmethylene amino) acetamides and 2-[2-(4chlorophenyl) benzimidazole-1-yl]-N-(4-oxo-2-arylthiazolidine-3-yl)acetamides-I. Chem Biol Drug Des 2012; 79: 869-877.

11. Kanagarajan V, Thanusu J, Gopalakrishnan M. Synthesis and in vitro microbiological evaluation of an array of biolabile 2-morpholino-N-(4,6-diarylpyrimidin-2yl)acetamides. Eur J Med Chem 2010; 45: 1583-1589.

12. Kaspady M, Narayanaswamy VK, Raju M, Rao GK. Synthesis, antibacterial activity of 2,4-disubstituted oxazoles and thiazoles as bioesters. Lett Drug Des Discov 2009; 6: 21-28.

13. Yang CR, Zhang Y, Jacob MR, Khan SI, Zhang YJ, Li $X C$. Antifungal activity of $C-27$ steroidal saponins. Antimicrob Agents Chemother 2006; 50: 1710-1714.

14. Sharma $P$, Sharma JD. In vitro hemolysis of human erythrocytes by plant extracts with antiplasmodial activity. J Ethnopharmacol 2001; 74: 239-243.

15. Powell WA, Catranis CM, Maynard CA. Design of selfprocessing antimicrobial peptide for plant protection. Lett Appl Microbiol 2000; 31(2): 163-168. 